\title{
The milliarcsecond-scale jet of PKS 0735+178 during quiescence
}

\author{
I. Agudo ${ }^{1,2}$, J. L. Gómez ${ }^{2}$, D. C. Gabuzda ${ }^{3}$, A. P. Marscher ${ }^{4}$, S. G. Jorstad ${ }^{4,5}$, and A. Alberdi ${ }^{2}$ \\ 1 Max-Planck-Institut für Radioastronomie, Auf dem Hügel 69, 53121 Bonn, Germany \\ e-mail: iagudo@mpifr-bonn.mpg.de \\ 2 Instituto de Astrofísica de Andalucía, CSIC, Apartado 3004, 18080 Granada, Spain \\ 3 Physics Department, University College Cork, Cork, Ireland \\ 4 Institute for Astrophysical Research, Boston University, 725 Commonwealth Avenue, Boston, MA 02215, USA \\ 5 Sobolev Astronomical Institute, St. Petersburg State University, Universitetskij Pr. 28, 198504 St. Petersburg, Russia
}

Received 14 November 2005 / Accepted 30 January 2006

\section{ABSTRACT}

\begin{abstract}
We present polarimetric $5 \mathrm{GHz}$ to $43 \mathrm{GHz}$ VLBI observations of the BL Lacertae object PKS 0735+178, spanning March 1996 to May 2000. Comparison with previous and later observations suggests that the overall kinematic and structural properties of the jet are greatly influenced by its activity. Time intervals of enhanced activity, as reported before 1993 and after 2000 by other studies, are followed by highly superluminal motion along a rectilinear jet. In contrast the less active state in which we performed our observations, shows subluminal or slow superluminal jet features propagating through a twisted jet with two sharp bends of about $90^{\circ}$ within the innermost three-milliarcsecond jet structure. Proper motion estimates from the data presented here allow us to constrain the jet viewing angle to values $\leq 9^{\circ}$, and the bulk Lorentz factor to be between 2 and 4 .
\end{abstract}

Key words. galaxies: active - galaxies: jets - polarization - BL Lacertae objects: individual: PKS 0735+178 radio continuum: galaxies - techniques: interferometric

\section{Introduction}

PKS $0735+178(0735+178$ throughout $)$ was first identified as a BLLacertae object (BLLac) by Carswell et al. (1974). These authors reported absorption lines redshifted by $z_{\text {abs }}=$ 0.424 , which was later confirmed by Rector \& Stocke (2001). Given that only absorption lines were detected, this $z_{\text {abs }}$ should be considered as a lower limit for the redshift of $0735+178$. Throughout this paper we assume $z=z_{\mathrm{abs}}=$ 0.424 , a Hubble constant $H_{0}=72 \mathrm{~km} \mathrm{~s}^{-1} \mathrm{Mpc}^{-1}$ and a Friedmann-Robertson-Walker cosmology with $\Omega_{\mathrm{m}}=0.3$ and $\Omega_{\Lambda}=0.7$. Under these assumptions the luminosity distance of $0735+178$ is $d_{\mathrm{L}}=2263 \mathrm{Mpc}$, an angular size of one milliarcsecond (mas) on the plane of the sky corresponds to a linear size of $5.4 \mathrm{pc}$ in the frame of the source, and an angular motion of $1 \mathrm{mas} / \mathrm{yr}$ corresponds to a speed of $25.1 \mathrm{c}$.

At radio wavelengths, $0735+178$ is predominantly pointlike at arcsecond resolution (Ulvestad et al. 1983; Kollgaard et al. 1992). Radio images made from data obtained with early very long baseline interferometry (VLBI) arrays at intermediate centimetre wavelengths have typically shown a compact core and a rather straight jet extending toward the north-east (e.g., Gabuzda et al. 1994). Multi-epoch VLBI observations made before 1993 allowed the identification of a number of superluminal jet features, with apparent speeds in the range $7 c$ to $12 c$ (Gabuzda et al. 1994, and references therein).

After 1994, VLBI observations at higher frequencies provided evidence for a curved structure within the inner jet of 0735+178 (Kellermann et al. 1998; Gómez et al. 1999). In particular, the polarimetric $22 \mathrm{GHz}$ and $43 \mathrm{GHz}$ images of Gómez et al. (1999), obtained in late 1996, revealed a twisted jet with two sharp apparent bends within the innermost 3 mas. Throughout the rest of the paper, we will refer to these two bends as the first ( $\sim 1$ mas east of the core, where the jet turns toward the north) and second ( $\sim 2$ mas north-east of the core, where the jet turns toward the east) bends. Gómez et al. (1999) also found the polarized emission following the outermost bend in the jet, with the magnetic vector being parallel to the local jet axis. Ojha et al. (2004) showed a very similar $15 \mathrm{GHz}$ polarization structure for a nearby epoch. Based on $8.4 \mathrm{GHz}$ and $43 \mathrm{GHz}$ VLBI observations spanning from 1995 to 1998 , along with existing previous observations, Gómez et al. (2001) studied the kinematics of the components since the first VLBI observations of $0735+178$, made in 1979. Their results raised the possibility that the jet proper motions for epochs after 1994 could be much slower than measured before 1993.

Comparison of $15 \mathrm{GHz}$ VLBI observations in February 1999 and $5 \mathrm{GHz} \mathrm{VSOP}^{1}$ observations in January 1999, both probing the same jet angular scales, showed markedly different jet structures at these two frequencies (Gabuzda et al. 2001). The most striking difference was the relative weakness of the $5 \mathrm{GHz}$ emission near the first bend, which implied a highly inverted spectrum in this area. This behaviour was shown to be consistent with free-free absorption of the emission near this bend. Evidence for a local enhancement in the Faraday rotation measure (RM) of $(120 \pm 55) \mathrm{rad} / \mathrm{m}^{2}$ was found for the same region. This behaviour was interpreted as being consistent with an interaction of the jet with the external medium surrounding the region of the first sharp bend, similar to the situation observed for the VLBI jet of the radio galaxy 3C 120 (Gómez et al. 2000).

Here we present polarimetric $5 \mathrm{GHz}$ to $43 \mathrm{GHz}$ VLBI observations of 0735+178, spanning from March 1996 to May 2000. This allows us to trace the magnetic field pattern where the jet executes the two bends. We also carry out a more complete study

\footnotetext{
${ }^{1}$ VLBI Space Observatory Program.
} 
Table 1. Observing epochs and frequencies.

\begin{tabular}{lcc}
\hline \hline Epoch & Date & Freq. (GHz) \\
\hline 1996.23 & 26 March 1996 & $8.4,22$ \\
1996.75 & 2 October 1996 & $8.4,22$ \\
1997.26 & 6 April 1997 & $5,8.4,15,22$ \\
1997.80 & 18 October 1997 & $8.4,22$ \\
1999.16 & 27 February 1999 & $15,22,43$ \\
1999.67 & 1 September 1999 & $15,22,43$ \\
2000.39 & 20 May 2000 & $15,22,43$ \\
\hline
\end{tabular}

of proper motions and changes in intensity along the jet than has been possible in earlier studies.

\section{Observations and data reduction}

The new polarimetric observations presented in this paper were obtained within two observing programs, both using the Very Long Baseline Array (VLBA) of the National Radio Astronomy Observatory $\left(\mathrm{NRAO}^{2}\right)$. The first consisted of four observing sessions of $12 \mathrm{~h}$ performed on 26 March 1996, 2 October 1996, 6 April 1997 and 18 October 1997 at $8.4 \mathrm{GHz}$ and $22 \mathrm{GHz}$. During the 6 April 1997 observations, $5 \mathrm{GHz}$ and $15 \mathrm{GHz}$ were also measured, see Table 1 . The second observing program consisted of three observing sessions of $12 \mathrm{~h}$ at $15 \mathrm{GHz}, 22 \mathrm{GHz}$ and $43 \mathrm{GHz}$ performed on 27 February 1999, 1 September 1999 and 20 May 2000. The $15 \mathrm{GHz}$ image from February 1999 was used for spectral index and RM analysis by Gabuzda et al. (2001).

The total-integration times on $0735+178$ were about $40 \mathrm{~min}$ at $5 \mathrm{GHz}, 60 \mathrm{~min}$ at $8.4 \mathrm{GHz}, 75 \mathrm{~min}$ at $15 \mathrm{GHz}, 95 \mathrm{~min}$ at $22 \mathrm{GHz}$ and $230 \mathrm{~min}$ at $43 \mathrm{GHz}$.

Both left- and right-circular polarization data were recorded at each of the VLBA telescopes at $128 \mathrm{Mbps}$, with 1 bit sampling and eight IFs each of $8 \mathrm{MHz}$ bandwidth. The data were correlated at the VLBA correlator in Socorro, New Mexico. The calibration of the data was performed with the AIPS package (Fomalont 1981) following the standard procedure for polarimetric observations (e.g. Leppänen et al. 1995). Opacity corrections for the $22 \mathrm{GHz}$ and $43 \mathrm{GHz}$ data were performed using the variation of the system temperature with elevation and solving for the receiver temperature and zenith opacity at each of the VLBA antennas. Opacity corrections for the $15 \mathrm{GHz}$ data were negligible. The instrumental polarization terms were determined using the parallactic angle dependence to separate the source polarization and the instrumental polarization through the feed-solution algorithm developed by Leppänen et al. (1995).

After the initial reduction, the data were edited, selfcalibrated, imaged and deconvolved using CLEAN both in total intensity $(I)$ and polarization $(P)$ using a combination of AIPS and the Caltech DIFMAP software (Pearson et al. 1994).

Calibration of the absolute orientation of the polarization position angles $(\chi)$ was performed by comparing the $\chi$ values corresponding to the total polarization in the VLBA images for $0735+178$ and various calibrators and other target sources (PKS 0420-014, PKS 0528+134, OJ 287, 3C 279, 3C 454.3 and BLLac) with those measured with the VLA at contemporaneous epochs. We also used the data from the VLA/VLBA Polarization Calibration service ${ }^{3}$ and data from the $4.8 \mathrm{GHz}$.

\footnotetext{
2 The NRAO of the USA is operated by Associated Universities, Inc., under co-operative agreement with the National Science Foundation.

3 http://www.aoc.nrao.edu/ smyers/calibration/.
}

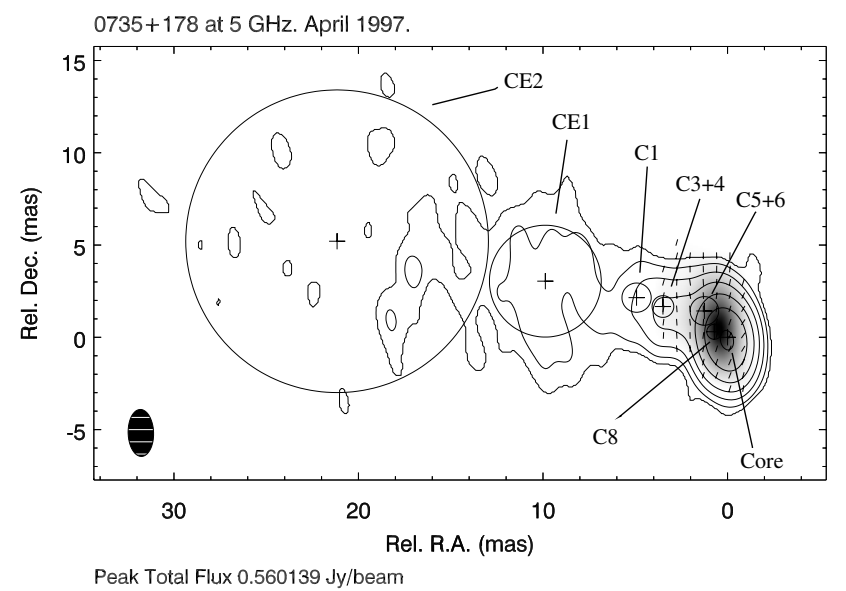

Total Flux Contours 0.25,0.67,1.78,4.74,12.65,33.74,90\% Beam FWHM $2.54725 \times 1.37742$ mas at $1.77175 \mathrm{deg}$.

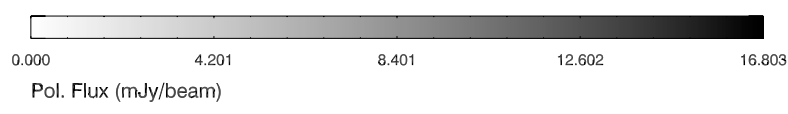

Fig. 1. $5 \mathrm{GHz}$ VLBA image of $0735+178$ on 6 April 1997. The contours represent the observed total intensity, the grey scale the polarized intensity and the superimposed sticks show the orientation of the polarization electric vectors. The positions of the fitted Gaussian components are indicated by the crosses, whereas the circles (of radius equal to the FWHM of each Gaussian) symbolize their size.

$8 \mathrm{GHz}$ and $14.5 \mathrm{GHz} \mathrm{UMRAO}^{4}$ monitoring program as a crosscheck. The typical uncertainties in the $\chi$ values range between $3^{\circ}$ and $5^{\circ}$ at all frequencies below $43 \mathrm{GHz}$; at the single epoch for which we detected polarized emission in $0735+178$ at $43 \mathrm{GHz}$ (May 2000), the uncertainty was larger, $\sim 7^{\circ}$.

\section{Results}

The resulting $I$ and $P$ images at $5 \mathrm{GHz}, 8.4 \mathrm{GHz}, 15 \mathrm{GHz}$, $22 \mathrm{GHz}$ and $43 \mathrm{GHz}$ are presented in Figs. 1-5. The I images were obtained using uniformly weighted $(u, v)$-coverage, whereas the $P$ (Stokes $Q$ and $U$ ) images were obtained using natural weighting.

The frequency coverage of our observations allowed us to study each region of the jet with adequate resolution within the inner 10 mas. The bends in the inner jet are not clearly visible at $5 \mathrm{GHz}$ (Fig. 1) due to the relatively low angular resolution and the weakness of the emission near the first sharp bend at this frequency (due to low-frequency absorption, as reported by Gabuzda et al. 2001). The VLBI structure at $5 \mathrm{GHz}$ is dominated by a compact core plus a broad jet extended toward the north-east up to $\sim 20$ mas to $\sim 30$ mas from the core. At the other frequencies, the two previously reported sharp bends of $\sim 90^{\circ}$ can be seen within the inner 3 mas of the jet (Figs. 2-4). Comparison of our new $8.4 \mathrm{GHz}, 15 \mathrm{GHz}$ and $22 \mathrm{GHz}$ images and previous I images (Kellermann et al. 1998; Gómez et al. 1999, 2001; Gabuzda et al. 2001) shows that the source has maintained its double bend structure from 1996 to 2000 . However, in the last three epochs, the second bend seems to decrease its curvature slightly (see Fig. 3).

The $5 \mathrm{GHz}$ and $8.4 \mathrm{GHz}$ maps (Figs. 1 and 2) show the polarization structure extending to $\sim 5$ mas from the core. As was

\footnotetext{
4 The University of Michigan Radio Astronomy Observatory is supported by funds provided by the University of Michigan.
} 


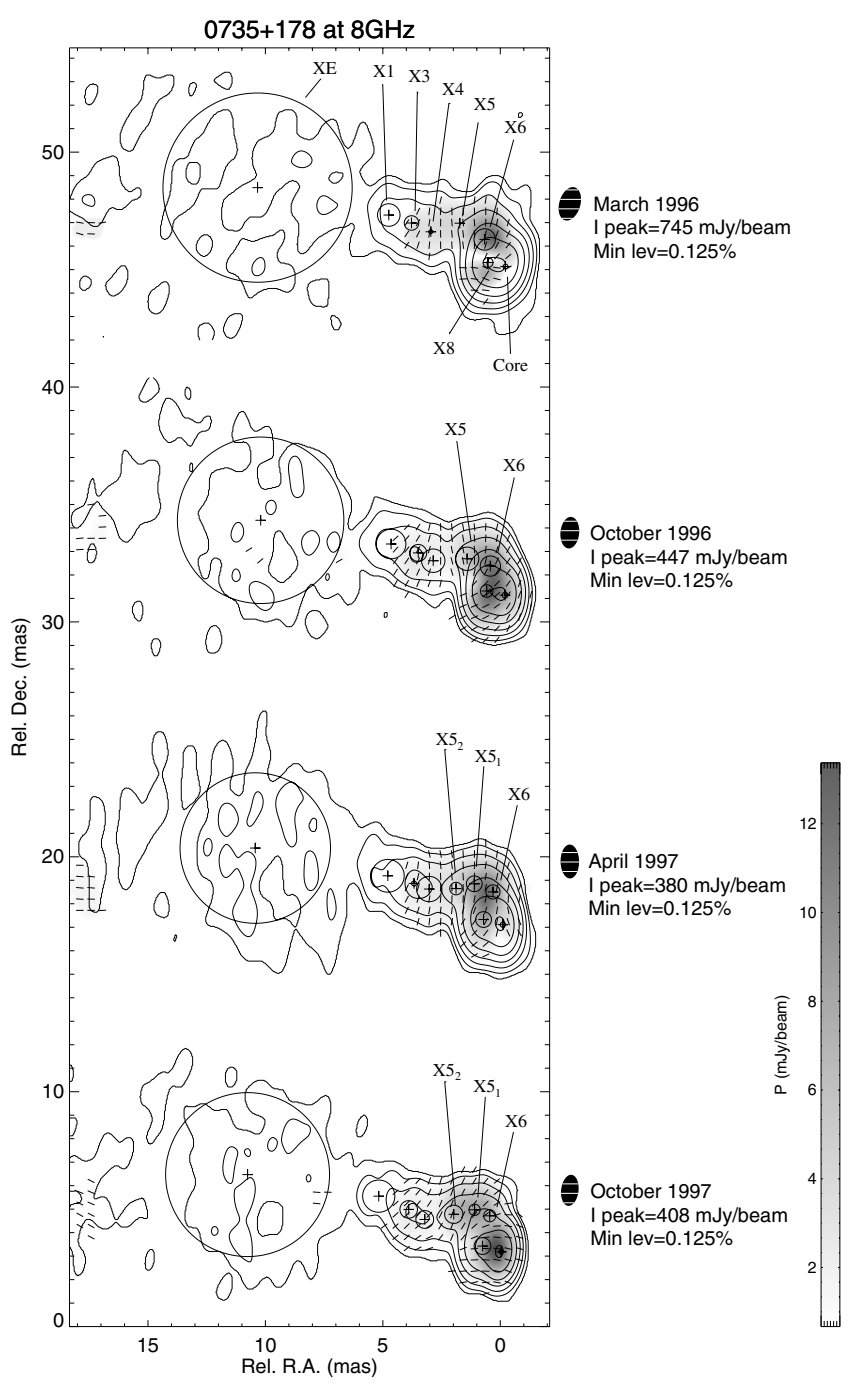

Fig. 2. 8.4 GHz VLBA images of $0735+178$. Observing epochs are given for each image. The total intensity is plotted as contours at seven logarithmically equispaced levels, starting at the minimum (indicated to the right of each image) up to the $90 \%$ of the corresponding I peak (also given for each map). The colour scale shows the distribution of the polarized intensity and the superimposed sticks show the orientation of the polarization electric vectors. From top to bottom, the convolving beams (shown as filled ellipses) are $(1.46 \times 0.90) \mathrm{mas}^{2},(1.35 \times$ $0.80) \mathrm{mas}^{2},(1.44 \times 0.79) \mathrm{mas}^{2}$ and $(1.33 \times 0.74)$ mas $^{2}$ with major-axis position angles at $-16.6^{\circ},-5.4^{\circ}, 0.6^{\circ}$ and $-6.5^{\circ}$, respectively. The positions of the fitted Gaussian components are indicated by the crosses, whereas the circles (of radius equal to the FWHM of each Gaussian) symbolize their size.

also reported from previous VLBI observations, the $\chi$ values outside the first bend are aligned perpendicularly to the local jet axis (see e.g. Figs. 2 and 3). The region of the first bend, and inwards, displays a high degree of variation in polarization between our observing epochs (Figs. 2 and 3).

\subsection{Jet structure modelling}

To describe the total intensity distribution in the $0735+178$ jet, we derived circular Gaussian model fits to all the data sets, in the visibility plane, using the DIFMAP software. The resulting parameters of the model fits, as well as the electric-vector position angle $\chi$ and degree of polarization $(m)$, computed as the $\chi$ and

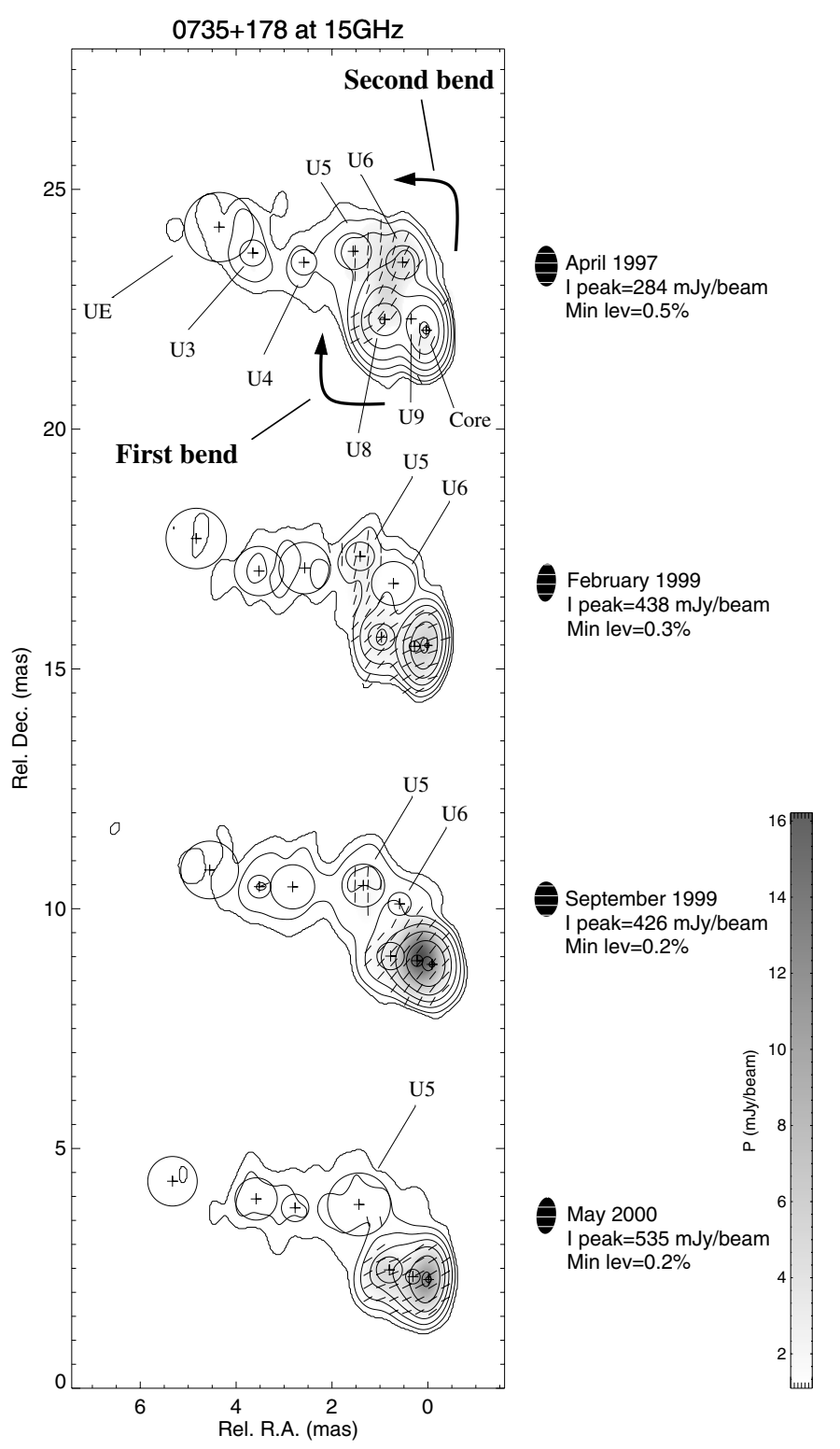

Fig. 3. Same as Fig. 2 for the $15 \mathrm{GHz}$ images. The location of the first and second bends defined in Sect. 1 are indicated in the image taken on April 1997. From top to bottom, the convolving beams are $(0.85 \times 0.47) \mathrm{mas}^{2},(0.80 \times 0.40) \mathrm{mas}^{2},(0.73 \times 0.49) \mathrm{mas}^{2}$ and $(0.76 \times 0.41)$ mas $^{2}$ with major axis position angles at $0.5^{\circ},-4.7^{\circ}, 1.1^{\circ}$ and $-4.8^{\circ}$, respectively.

$m$ averages in boxes of $3 \times 3$ pixels around the position of each component, are listed in Tables 2 to 6 .

Note that a slightly different $15 \mathrm{GHz}$ model fit than that presented by Gabuzda et al. (2001) is presented here for February 1999 (Table 4). The new model contains an extra component in the region between 3 mas and 4 mas from the core. The reason for changing this model was to make it more consistent with the modelling of this region at $15 \mathrm{GHz}$ for the remaining observing epochs by considering the same number of components across epochs. Note, however, that this does not affect in any way the validity of the results presented by Gabuzda et al. (2001).

To obtain a good representation of the jet structure at $22 \mathrm{GHz}$ (epochs March 1996 and October 1996) and $43 \mathrm{GHz}$ (epochs February 1999, September 1999 and May 2000) it was necessary to split the emission regions corresponding to components $i 8$ 


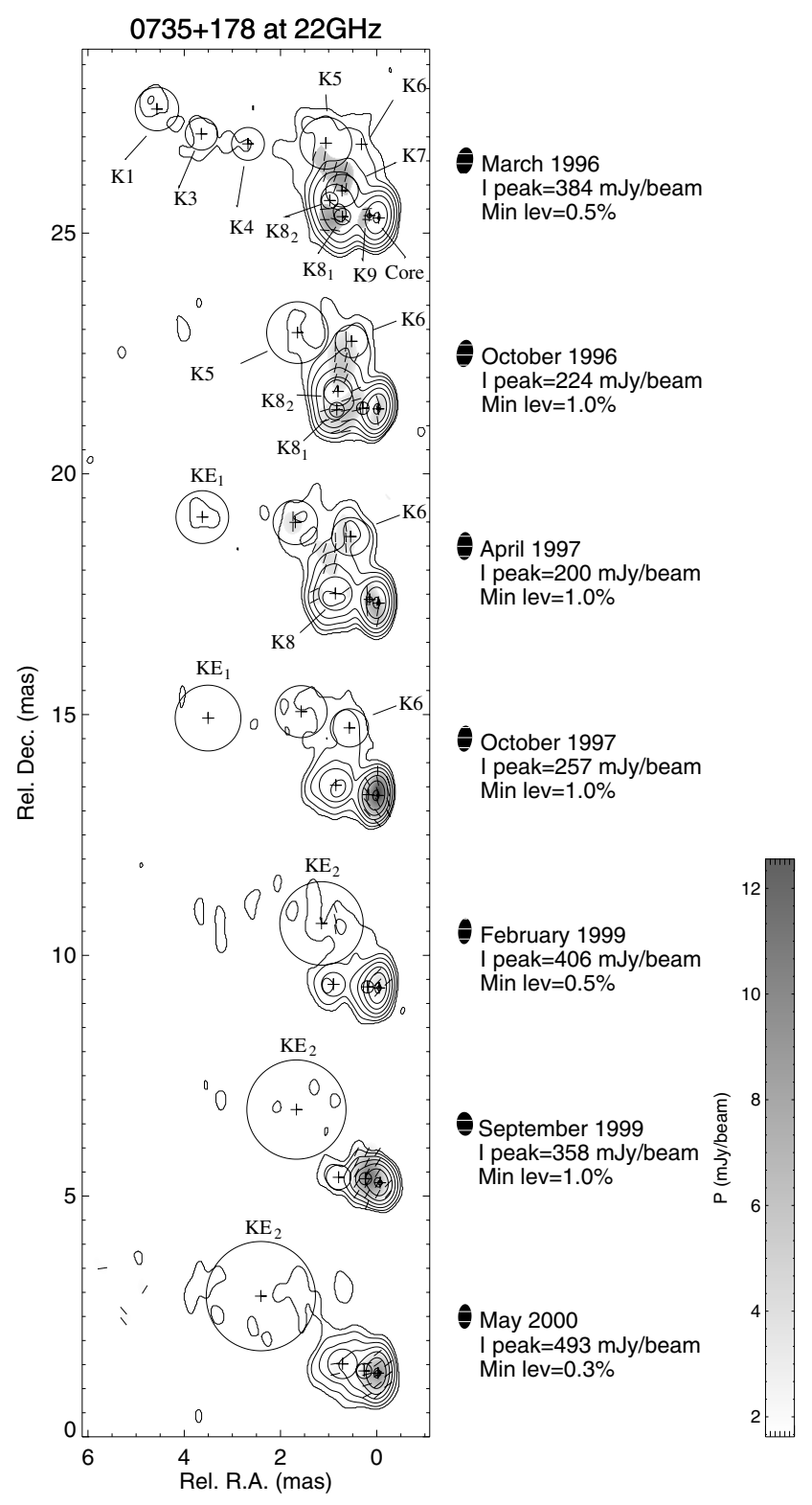

Fig. 4. Same as Fig. 2 but for the $22 \mathrm{GHz}$ images. From top to bottom, the convolving beams are $(0.57 \times 0.34) \mathrm{mas}^{2},(0.57 \times 0.34) \mathrm{mas}^{2}$, $(0.58 \times 0.32) \mathrm{mas}^{2},(0.54 \times 0.30) \mathrm{mas}^{2},(0.54 \times 0.28) \mathrm{mas}^{2},(0.49 \times$ $0.34) \mathrm{mas}^{2}$ and $(0.51 \times 0.28) \mathrm{mas}^{2}$ with major axis position angles at $-9.5^{\circ},-9.6^{\circ},-3.0^{\circ},-6.0^{\circ},-4.7^{\circ}, 5.4^{\circ}$ and $-2.0^{\circ}$, respectively.

and $i 9$ (with $i=\mathrm{C}, \mathrm{X}, \mathrm{U}$; see Figs. 1 to 5 and Tables 2 to 6 ) into several sub-components, labelled $\mathrm{K} 8_{1}, \mathrm{~K} 8_{2}, \mathrm{Q} 8_{1}, \mathrm{Q} 8_{2}, \mathrm{Q} 8_{3}$, Q9 ${ }_{1}$ and $\mathrm{Q} 9_{2}$. For a better characterization of all the jet components it was also necessary to fit the extended emission observed in the outer regions of the jet (modelled as components $i \mathrm{E}$ with $i=\mathrm{C}, \mathrm{X}, \mathrm{U}, \mathrm{K}$; see Figs. 1 to 4 and Tables 2 to 5). Note that these components should not be taken as representing real distinct features in the jet, but instead extended regions with weak flux densities.

The errors in the DIFMAP model-fit parameters (total flux density, position and size of the components) were obtained using the DIFWRAP software, developed by Lovell (2000). The errors were determined by perturbing the fit parameters until the resulting residual maps were unacceptable. These perturbed quantities defined the upper and lower limits for the errors of the fit parameters. To take into account the interrelation

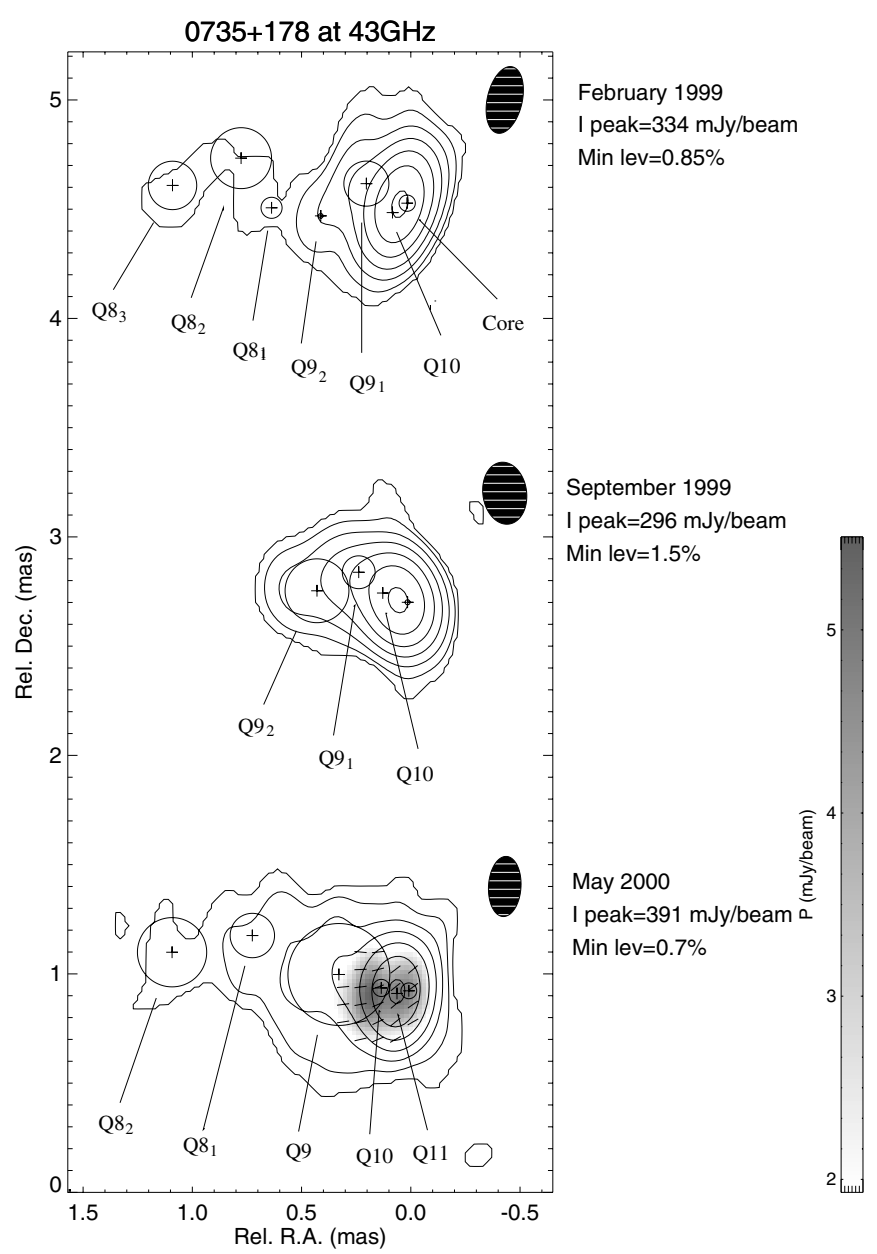

Fig. 5. Same as Fig. 2 but for the $43 \mathrm{GHz}$ images. From top to bottom, the convolving beams are $(0.31 \times 0.16) \mathrm{mas}^{2},(0.29 \times 0.20) \mathrm{mas}^{2}$ and $(0.28 \times 0.15)$ mas $^{2}$ with major axis position angles at $-12.8^{\circ}, 7.8^{\circ}$ and $-3.4^{\circ}$, respectively.

between the fit parameters, we also allowed for simultaneous variations of all four parameters for each component when determining the final uncertainties. This resulted in somewhat larger but more conservative error estimates (see Lovell 2000, and http: //www.vsop. isas.ac. jp/survey/di fwrap).

The $\chi$ errors were computed from the uncertainties in the $\chi$ calibration for each map added in quadrature to the rms noise within the $3 \times 3$ pixel box in which $\chi$ was averaged. The $m$ uncertainties were obtained as the propagated errors from the $I$ and $P$ measurements within the averaging box, for which the rms noise of $I$ and $P$ was taken as uncertainties of their corresponding measurements.

\section{Discussion}

\subsection{Kinematics of model components}

Figure 6 represents the positions of all the model components in Tables 2 to 6 within the inner 6 mas of the jet for which reliable position estimates were obtained. This figure shows a clustering of components around some jet locations, marked by dashed circles. This clustering was rather stable during a period of four years and is very unlikely to be produced by chance. This kind of component distribution was also shown by Gómez et al. (2001) for a different series of data spanning from 1995 to 1998 . The clustering implies a much slower scenario than 
Table 2. 5 GHz model fits for 6 April 1997.

\begin{tabular}{lcccccc}
\hline \hline Comp. & $\begin{array}{c}I \\
(\mathrm{mJy})\end{array}$ & $\begin{array}{c}r \\
(\mathrm{mas})\end{array}$ & $\begin{array}{c}\theta \\
\left.{ }^{\circ}\right)\end{array}$ & $\begin{array}{c}F W H M \\
(\mathrm{mas})\end{array}$ & $\begin{array}{c}\chi \\
\left.{ }^{\circ}\right)\end{array}$ & $\begin{array}{c}m \\
\%\end{array}$ \\
\hline Core & $249 \pm 12$ & 0.00 & 0 & $<0.43$ & $-23 \pm 3$ & $2.2 \pm 0.3$ \\
$\mathrm{C} 8$ & $455 \pm 8$ & $0.78 \pm 0.09$ & $67 \pm 30$ & $0.82 \pm 0.08$ & $-14 \pm 3$ & $4.1 \pm 0.4$ \\
$\mathrm{C} 5+6$ & $100 \pm 13$ & $1.90 \pm 0.15$ & $41 \pm 5$ & $1.55 \pm 0.23$ & $-1 \pm 3$ & $10 \pm 2$ \\
$\mathrm{C} 3+4$ & $47 \pm 11$ & $3.88 \pm 0.15$ & $65 \pm 6$ & $1.18 \pm 0.51$ & $-11 \pm 4$ & $8 \pm 5$ \\
$\mathrm{C} 1$ & $19 \pm 9$ & $5.38 \pm 0.60$ & $66 \pm 10$ & $1.59 \pm 0.93$ & $\ldots$ & $\ldots$ \\
$\mathrm{CE} 1$ & $70 \pm 40$ & $10.33 \pm 1.19$ & $73 \pm 6$ & $6.06 \pm 1.67$ & $\ldots$ & $\ldots$ \\
$\mathrm{CE} 2$ & $\sim 81$ & $\sim 21.79$ & $\sim 76$ & $\sim 16.40$ & $\ldots$ & $\ldots$ \\
\hline
\end{tabular}

reported for epochs before 1993 (Gabuzda et al. 1994, and references therein), with proper motions in the range $0.28{\text { mas } \mathrm{yr}^{-1}}^{-1}$ (7c) to 0.48 mas $r^{-1}(12 c)$. Such proper motions would be observed as systematic shifts of the positions of the model components by 1.12 mas to 1.92 mas during the time range of our monitoring ( $\sim 4 \mathrm{yr})$, which are not observed in any of the jet regions.

Figure 7 presents the distance from the core as a function of time for the innermost (and most reliable) $8.4 \mathrm{GHz}, 15 \mathrm{GHz}$, $22 \mathrm{GHz}$ and $43 \mathrm{GHz}$ model components in Tables 2 to 6 and those published by Gómez et al. (2001). The $5 \mathrm{GHz}$ data in Table 2 are not plotted due to the much lower resolution at this frequency, which can produce confusion in the identification of components. The evidence of slow proper motions along the jet allowed us to ensure the most reliable identification of components as the one which relates a jet feature with those nearest in position at different observing frequencies and at adjacent observing epochs.

It is worth to note the significant discrepancy between the identification of components presented here and the one performed by Kellermann et al. (2004) through their quasi-annual $15 \mathrm{GHz}$ observations of $0735+178$ from 1995 to 2000 . This discrepancy is most probably caused by the poorer time and frequency sampling of Kellermann et al. and also by the different model-fitting method used by them, which only considered a maximum of three jet components per epoch and was performed in the image plane. Figure 3 shows that a set of only three jet components does not suffice to optimally fit the whole VLBI structure of $0735+178$ at $15 \mathrm{GHz}$.

Our fits of the radial positions of components $i 8, i 9$ and $i 10$ show slow radial motions outward from the core with speeds of $(0.023 \pm 0.004)$ mas $^{-1} r^{-1},(0.048 \pm 0.004)$ mas $\mathrm{yr}^{-1}$ and $(0.026 \pm$

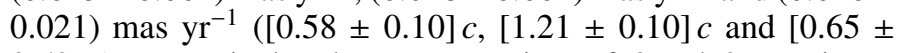
$0.53] c$ ), respectively. The proper motions of $i 8$ and $i 9$ were interpreted as consistent with a quasi-stationary behaviour by Gómez et al. (2001) for a shorter time range (from 1995 to 1998) than the one covered by our observations. Figure 6 shows that these two model components are more consistently fitted as moving features if our last three observing epochs (from 1999 to 2000) are taken into account. This implies that both $i 8$ and $i 9$ slightly speeded up after 1998, which is more evident for $i 9$ (Fig. 6). It should also be noted that our estimates provide only the speeds of the average position of the components during our observations. The unaccelerated proper motion of $0.14 \pm 0.03$ mas $\mathrm{yr}^{-1}$ estimated by Homan et al. (2001) for $i 8$, through their bimonthly monitoring program during 1996, accounts for the irregular motion of this component around its average position. This rapid, but short distance, motion explains the clumping of components around their mean positions, which are most likely produced by the changes of their brightness distributions.

The fits for components $i 1, i 3$ and $i 4$ show slight upstream radial motions. Although these motions could, in principle,
Table 3. $8.4 \mathrm{GHz}$ model fits.

\begin{tabular}{|c|c|c|c|c|c|c|}
\hline Comp. & $\begin{array}{c}I \\
(\mathrm{mJy})\end{array}$ & $\begin{array}{c}r \\
\text { (mas) }\end{array}$ & $\begin{array}{c}\theta \\
\left({ }^{\circ}\right)\end{array}$ & $\begin{array}{c}F W H M \\
\text { (mas) }\end{array}$ & $\begin{array}{c}\chi \\
\left(^{\circ}\right)\end{array}$ & $\begin{array}{l}m \\
\%\end{array}$ \\
\hline \multicolumn{7}{|c|}{26 March 1996} \\
\hline Core & $590 \pm 39$ & 0.00 & 0 & $0.20 \pm 0.11$ & $\ldots$ & $\ldots$ \\
\hline $\mathrm{X} 8$ & $629 \pm 24$ & $0.76 \pm 0.01$ & $77 \pm 2$ & $0.42 \pm 0.06$ & $\ldots$ & $\ldots$ \\
\hline X6 & $161 \pm 24$ & $1.45 \pm 0.02$ & $38 \pm 2$ & $0.91 \pm 0.22$ & $-0.8 \pm 3$ & $7 \pm 1$ \\
\hline $\mathrm{X} 5$ & $16 \pm 9$ & $2.68 \pm 0.27$ & $47 \pm 5$ & $<0.54$ & $5 \pm 3$ & $23 \pm 11$ \\
\hline $\mathrm{X} 4$ & $23 \pm 12$ & $3.51 \pm 0.24$ & $65 \pm 9$ & $<0.61$ & $-16 \pm 3$ & $14 \pm 8$ \\
\hline $\mathrm{X} 3$ & $21 \pm 15$ & $4.41 \pm 0.47$ & $65 \pm 8$ & $<1.21$ & $\ldots$ & $\ldots$ \\
\hline $\mathrm{X} 1$ & $20 \pm 15$ & $5.43 \pm 0.66$ & $66 \pm 13$ & $<2.10$ & $\ldots$ & $\ldots$ \\
\hline $\mathrm{XE}$ & $\sim 77$ & $\sim 11.08$ & $\sim 72$ & $\sim 8.06$ & $\ldots$ & $\ldots$ \\
\hline \multicolumn{7}{|c|}{2 October 1996} \\
\hline Core & $396 \pm 4$ & 0.00 & 0 & $0.25 \pm 0.04$ & $-47 \pm 3$ & $2.4 \pm 0.2$ \\
\hline $\mathrm{X} 8$ & $434 \pm 6$ & $0.80 \pm 0.01$ & $77 \pm 1$ & $0.53 \pm 0.02$ & $-49 \pm 3$ & $2.7 \pm 0.3$ \\
\hline X6 & $110 \pm 11$ & $1.39 \pm 0.02$ & $27 \pm 1$ & $0.86 \pm 0.03$ & $-15 \pm 3$ & $8.6 \pm 0.8$ \\
\hline $\mathrm{X} 5$ & $36 \pm 8$ & $2.24 \pm 0.10$ & $46 \pm 2$ & $0.99 \pm 0.15$ & $13 \pm 3$ & $25 \pm 5$ \\
\hline $\mathrm{X} 4$ & $15 \pm 8$ & $3.39 \pm 0.24$ & $64 \pm 7$ & $1.00 \pm 0.43$ & $-21 \pm 3$ & $19 \pm 6$ \\
\hline $\mathrm{X} 3$ & $18 \pm 8$ & $4.11 \pm 0.16$ & $64 \pm 4$ & $0.72 \pm 0.43$ & $-36 \pm 3$ & $17 \pm 6$ \\
\hline $\mathrm{X} 1$ & $19 \pm 12$ & $5.32 \pm 0.35$ & $66 \pm 6$ & $1.26 \pm 0.53$ & $\ldots$ & $\ldots$ \\
\hline $\mathrm{XE}$ & $\sim 62$ & $\sim 10.8$ & $\sim 73$ & $\sim 7.09$ & $\ldots$ & $\ldots$ \\
\hline \multicolumn{7}{|c|}{6 April 1997} \\
\hline Core & $350 \pm 4$ & 0.00 & 0 & $0.22 \pm 0.03$ & $-3 \pm 4$ & $1.1 \pm 0.3$ \\
\hline $\mathrm{X} 8$ & $310 \pm 6$ & $0.85 \pm 0.01$ & $75 \pm 1$ & $0.67 \pm 0.02$ & $-25 \pm 3$ & $3.9 \pm 0.6$ \\
\hline X6 & $52 \pm 8$ & $1.46 \pm 0.05$ & $17 \pm 2$ & $0.60 \pm 0.10$ & $-11 \pm 3$ & $15.8 \pm 1.7$ \\
\hline $\mathrm{X} 5_{1}$ & $31 \pm 7$ & $2.12 \pm 0.08$ & $35 \pm 2$ & $0.67 \pm 0.15$ & $1 \pm 3$ & $21 \pm 4$ \\
\hline$X 5_{2}$ & $19 \pm 5$ & $2.52 \pm 0.09$ & $52 \pm 3$ & $0.53 \pm 0.34$ & $2 \pm 3$ & $19 \pm 6$ \\
\hline $\mathrm{X} 4$ & $23 \pm 7$ & $3.48 \pm 0.12$ & $64 \pm 3$ & $1.08 \pm 0.26$ & $-11 \pm 3$ & $17 \pm 7$ \\
\hline $\mathrm{X} 3$ & $12 \pm 4$ & $4.17 \pm 0.12$ & $65 \pm 2$ & $<0.56$ & $-23 \pm 3$ & $14 \pm 8$ \\
\hline $\mathrm{X} 1$ & $17 \pm 8$ & $5.32 \pm 0.25$ & $67 \pm 5$ & $1.39 \pm 0.73$ & $\ldots$ & $\ldots$ \\
\hline $\mathrm{XE}$ & $\sim 56$ & $\sim 11.04$ & $\sim 73$ & $\sim 6.41$ & $\ldots$ & $\ldots$ \\
\hline \multicolumn{7}{|c|}{18 October 1997} \\
\hline Core & $391 \pm 2$ & 0.00 & 0 & $0.18 \pm 0.01$ & $80 \pm 3$ & $3.2 \pm 0.2$ \\
\hline $\mathrm{X} 8$ & $222 \pm 2$ & $0.83 \pm 0.01$ & $73 \pm 1$ & $0.71 \pm 0.03$ & $-84 \pm 3$ & $4.9 \pm 0.5$ \\
\hline X6 & $40 \pm 4$ & $1.61 \pm 0.05$ & $18 \pm 1$ & $0.51 \pm 0.18$ & $-52 \pm 3$ & $14.5 \pm 1.4$ \\
\hline $\mathrm{X} 5_{1}$ & $28 \pm 4$ & $2.11 \pm 0.10$ & $32 \pm 2$ & $0.48 \pm 0.23$ & $-34 \pm 3$ & $24.3 \pm 2.2$ \\
\hline $\mathrm{X} 5_{2}$ & $37 \pm 4$ & $2.57 \pm 0.09$ & $52 \pm 2$ & $0.78 \pm 0.24$ & $-20 \pm 3$ & $23 \pm 3$ \\
\hline $\mathrm{X} 4$ & $18 \pm 7$ & $3.53 \pm 0.17$ & $67 \pm 4$ & $0.76 \pm 0.44$ & $-44 \pm 3$ & $14 \pm 5$ \\
\hline $\mathrm{X} 3$ & $18 \pm 6$ & $4.33 \pm 0.17$ & $65 \pm 3$ & $0.75 \pm 0.44$ & $-47 \pm 3$ & $16 \pm 5$ \\
\hline $\mathrm{X} 1$ & $10 \pm 9$ & $5.72 \pm 0.67$ & $66 \pm 9$ & $1.37 \pm 0.64$ & $\ldots$ & $\ldots$ \\
\hline $\mathrm{XE}$ & $\sim 67$ & $\sim 11.28$ & $\sim 73$ & $\sim 6.98$ & $\ldots$ & $\ldots$ \\
\hline
\end{tabular}

happen in actual relativistic jets under certain conditions (see e.g. Agudo et al. 2001), we prefer to consider these three components to be quasi-stationary in view of the large uncertainties in their positions and apparent motions (with values of

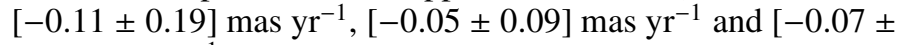

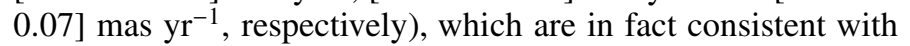
no motion. Further evidence for considering these components as quasi-stationary is based on their stable structural position angles, with average values of $66^{\circ} \pm 7^{\circ}, 65^{\circ} \pm 5^{\circ}$ and $64^{\circ} \pm 5^{\circ}$, respectively, and their stable total flux densities (see Sect. 4.2).

The behaviour of the components related to the second bend, $i 5$ and $i 6$, is very different. Inspection of Fig. 3 reveals a smooth change in the region of the second bend, which progressively softens from 1997 to 2000. Figure 6 also shows a significant dispersion for the position of component $i 6$ (from March 1996 to October 1997), which is larger than its typical position uncertainty (especially at $8.4 \mathrm{GHz}$ ). Figure 8 represents the structural position angles of components $i 5, \mathrm{X} 5_{1}$ and $i 6$ during the time covered by our observations. We can see that $i 6$ moved from a structural position angle of $\sim 38^{\circ}$ at the beginning of 1996 to $\sim 20^{\circ}$ from the end of 1996 to the beginning of 1997. During 1999 , it returned to $\sim 30^{\circ}$, then finally became undetectable in our last observing epoch (May 2000). Although the 
Table 4. $15 \mathrm{GHz}$ model fits.

\begin{tabular}{|c|c|c|c|c|c|c|}
\hline Comp. & $\begin{array}{c}I \\
(\mathrm{mJy}) \\
\end{array}$ & $\begin{array}{c}r \\
\text { (mas) }\end{array}$ & $\begin{array}{c}\theta \\
\left(^{\circ}\right) \\
\end{array}$ & $\begin{array}{c}F W H M \\
\text { (mas) }\end{array}$ & $\begin{array}{l}\chi \\
\left(^{\circ}\right) \\
\end{array}$ & $\begin{array}{l}m \\
\% \\
\end{array}$ \\
\hline \multicolumn{7}{|c|}{6 April 1997} \\
\hline Core & $292 \pm 7$ & 0.00 & 0 & $0.15 \pm 0.02$ & $-9 \pm 4$ & $0.6 \pm 0.5$ \\
\hline $\mathrm{U}^{1}$ & $15 \pm 8$ & $0.40 \pm 0.23$ & $53 \pm 23$ & $<0.38$ & $\ldots$ & $\ldots$ \\
\hline U8 & $236 \pm 18$ & $0.90 \pm 0.06$ & $75 \pm 2$ & $0.67 \pm 0.03$ & $-39 \pm 3$ & $2.6 \pm 1.3$ \\
\hline U6 & $47 \pm 9$ & $1.50 \pm 0.10$ & $19 \pm 2$ & $0.69 \pm 0.18$ & $-25 \pm 3$ & $18 \pm 7$ \\
\hline U5 & $24 \pm 12$ & $2.25 \pm 0.14$ & $43 \pm 4$ & $0.76 \pm 0.34$ & $-1 \pm 3$ & $22 \pm 15$ \\
\hline $\mathrm{U} 4^{1}$ & $10 \pm 10$ & $2.92 \pm 0.33$ & $61 \pm 8$ & $0.53 \pm 0.39$ & $\ldots$ & $\ldots$ \\
\hline $\mathrm{U} 3$ & $11 \pm 9$ & $3.96 \pm 0.31$ & $66 \pm 8$ & \pm 1.15 & $\ldots$ & $\ldots$ \\
\hline UE & $\sim 14$ & $\sim 4.83$ & $\sim 64$ & $\sim 1.45$ & $\ldots$ & $\ldots$ \\
\hline \multicolumn{7}{|c|}{ 27 February 1999} \\
\hline Core & $417 \pm 2$ & 0.00 & 0 & $0.09 \pm 0.02$ & $-69 \pm 3$ & $1.3 \pm 0.2$ \\
\hline U9 & $83 \pm 4$ & $0.26 \pm 0.01$ & $94 \pm 5$ & $0.22 \pm 0.13$ & $-68 \pm 3$ & $2.0 \pm 0.4$ \\
\hline U8 & $47 \pm 9$ & $0.96 \pm 0.05$ & $80 \pm 5$ & $0.55 \pm 0.18$ & $-46 \pm 3$ & $11 \pm 4$ \\
\hline U6 & $25 \pm 15$ & $1.47 \pm 0.25$ & $29 \pm 9$ & $0.91 \pm 0.32$ & $\ldots$ & $\ldots$ \\
\hline U5 & $13 \pm 9$ & $2.32 \pm 0.25$ & $37 \pm 6$ & $0.60 \pm 0.44$ & $-11 \pm 3$ & $36 \pm 17$ \\
\hline $\mathrm{U} 4$ & $18 \pm 13$ & $3.02 \pm 0.35$ & $58 \pm 9$ & $1.08 \pm 0.66$ & $\ldots$ & $\ldots$ \\
\hline $\mathrm{U} 3$ & $13 \pm 13$ & $3.83 \pm 0.44$ & $66 \pm 8$ & $1.03 \pm 1.01$ & $\ldots$ & $\ldots$ \\
\hline UE & $\sim 6$ & $\sim 5.31$ & $\sim 65$ & $\sim 1.26$ & $\ldots$ & $\ldots$ \\
\hline \multicolumn{7}{|c|}{ 1 September 1999} \\
\hline Core & $365 \pm 4$ & 0.00 & 0 & $0.12 \pm 0.03$ & $-38 \pm 5$ & $3.6 \pm 0.3$ \\
\hline U9 & $176 \pm 5$ & $0.32 \pm 0.01$ & $76 \pm 3$ & $0.24 \pm 0.07$ & $-39 \pm 5$ & $7.0 \pm 0.7$ \\
\hline U8 & $38 \pm 8$ & $0.88 \pm 0.06$ & $79 \pm 6$ & $0.57 \pm 0.16$ & $-44 \pm 5$ & $13 \pm 6$ \\
\hline U6 & $9 \pm 8$ & $1.43 \pm 0.39$ & $28 \pm 9$ & $0.48 \pm 0.44$ & $\ldots$ & $\ldots$ \\
\hline U5 & $24 \pm 9$ & $2.18 \pm 0.23$ & $41 \pm 6$ & $0.89 \pm 0.28$ & $-4 \pm 5$ & $21 \pm 18$ \\
\hline U4 & $17 \pm 10$ & $3.33 \pm 0.39$ & $61 \pm 6$ & $0.92 \pm 0.44$ & $\ldots$ & $\ldots$ \\
\hline U3 & $8 \pm 6$ & $3.95 \pm 0.39$ & $66 \pm 6$ & $<1.31$ & $\ldots$ & $\ldots$ \\
\hline UE & $\sim 6$ & $\sim 5.04$ & $\sim 67$ & $\sim 1.20$ & $\ldots$ & $\ldots$ \\
\hline \multicolumn{7}{|c|}{20 May 2000} \\
\hline Core & $523 \pm 6$ & 0.00 & 0 & $0.12 \pm 0.02$ & $-60 \pm 5$ & $2.0 \pm 0.2$ \\
\hline U9 & $144 \pm 9$ & $0.33 \pm 0.01$ & $79 \pm 7$ & $0.30 \pm 0.05$ & $-63 \pm 5$ & $2.9 \pm 0.6$ \\
\hline U8 & $86 \pm 8$ & $0.84 \pm 0.02$ & $76 \pm 3$ & $0.54 \pm 0.05$ & $-70 \pm 5$ & $10 \pm 3$ \\
\hline U5 & $30 \pm 17$ & $2.13 \pm 0.34$ & $43 \pm 15$ & $1.31 \pm 0.91$ & $\ldots$ & $\ldots$ \\
\hline U4 & $\sim 7$ & $\sim 3.16$ & $\sim 62$ & $\sim 0.57$ & $\ldots$ & $\ldots$ \\
\hline U3 & $\sim 15$ & $\sim 3.97$ & $\sim 66$ & $\sim 0.88$ & $\ldots$ & $\ldots$ \\
\hline UE & $\sim 3$ & $\sim 5.72$ & $\sim 69$ & $\sim 1.03$ & $\ldots$ & $\ldots$ \\
\hline
\end{tabular}

${ }^{1}$ Uncertain identification of this model component.

variations are less significant, the structural position angle of $i 5$ also changed from $\sim 47^{\circ}$ at the beginning of 1996 , to $\sim 52^{\circ}$, during 1997 , to $\sim 37^{\circ}$ at the beginning of 1999 , and then back to almost the initial position angle at the beginning of 2000. During the time period of maximum angular separation between i5 and $i 6$ (during 1997) a new component (X51) was fitted at intermediate angular positions of $35^{\circ} \pm 2^{\circ}$ (in April 1997) and $32^{\circ} \pm 2^{\circ}$ (in October 1997). Although it was necessary to introduce component $\mathrm{X} 5_{1}$ for these two epochs in order to obtain a good model fit, it seems very unlikely that this is related to the appearance of a new real feature in the jet. We consider $\mathrm{X} 5_{1}$ to represent emission from the underlying jet in the gap region produced as a consequence of the angular separation of $i 5$ and $i 6$.

The behaviour of $i 5$ and $i 6$ between 1996 and 2000 is related to a "softening" of the second bend, which is consistent with the fairly rectilinear east-north-east jet structure reported by the $15 \mathrm{GHz}$ image of Lister \& Homan (2005) taken on November 2002. This softening is produced by an apparent backward motion of component $i 6$. Again, this should not be understood as a physical motion of jet plasma back to the core, but rather as induced by a decrease in the total flux density of the bent area, shifting the peak of the emission (associated with the component) to positions closer to the core until component $i 6$ became undetectable by May 2000 (see Tables 2 to 6 and Sect. 4.2).
Table 5. $22 \mathrm{GHz}$ model fits.

\begin{tabular}{|c|c|c|c|c|c|c|}
\hline Comp. & $\begin{array}{c}I \\
(\mathrm{mJy})\end{array}$ & $\begin{array}{c}r \\
\text { (mas) }\end{array}$ & $\begin{array}{c}\theta \\
\left({ }^{\circ}\right)\end{array}$ & $\begin{array}{c}F W H M \\
(\text { mas })\end{array}$ & $\begin{array}{c}\chi \\
\left(^{\circ}\right)\end{array}$ & $\begin{array}{l}m \\
\%\end{array}$ \\
\hline \multicolumn{7}{|c|}{26 March 1996} \\
\hline Core & $353 \pm 9$ & 0.00 & 0 & $<0.10$ & $\ldots$ & $\ldots$ \\
\hline K9 & $73 \pm 9$ & $0.20 \pm 0.03$ & $76 \pm 18$ & $<0.32$ & $\ldots$ & $\ldots$ \\
\hline $\mathrm{K} 8_{1}$ & $232 \pm 13$ & $0.75 \pm 0.01$ & $88 \pm 1$ & $0.34 \pm 0.04$ & $60 \pm 4$ & $4 \pm 3$ \\
\hline K7 & $122 \pm 16$ & $0.95 \pm 0.05$ & $53 \pm 4$ & $0.68 \pm 0.10$ & $\ldots$ & $\ldots$ \\
\hline $\mathrm{K}_{2}$ & $93 \pm 13$ & $1.08 \pm 0.04$ & $70 \pm 3$ & $0.34 \pm 0.07$ & $\ldots$ & $\ldots$ \\
\hline K6 & $\sim 5$ & $\sim 1.57$ & $\sim 13$ & $\sim 0.01$ & $\ldots$ & $\ldots$ \\
\hline $\mathrm{K} 5^{1}$ & $45 \pm 34$ & $1.90 \pm 0.20$ & $35 \pm 8$ & $1.08 \pm 0.34$ & $\ldots$ & $\ldots$ \\
\hline K4 & $\sim 8$ & $\sim 3.12$ & $\sim 61$ & $\sim 0.68$ & $\ldots$ & $\ldots$ \\
\hline $\mathrm{K} 3$ & $\sim 10$ & $\sim 4.07$ & $\sim 65$ & $\sim 0.67$ & $\ldots$ & $\ldots$ \\
\hline K1 & $\sim 12$ & $\sim 5.13$ & $\sim 64$ & $\sim 0.90$ & $\ldots$ & $\ldots$ \\
\hline \multicolumn{7}{|c|}{2 October 1996} \\
\hline Core & $217 \pm 7$ & 0.00 & 0 & $<0.10$ & $14 \pm 3$ & $2.2 \pm 1.2$ \\
\hline K9 & $33 \pm 8$ & $0.32 \pm 0.05$ & $88 \pm 12$ & $0.26 \pm 0.05$ & $\ldots$ & $\ldots$ \\
\hline $\mathrm{K} 8_{1}$ & $70 \pm 10$ & $0.86 \pm 0.02$ & $91 \pm 2$ & $0.30 \pm 0.09$ & $-72 \pm 3$ & $6 \pm 4$ \\
\hline $\mathrm{K} 8_{2}$ & $153 \pm 10$ & $0.91 \pm 0.02$ & $67 \pm 3$ & $0.59 \pm 0.08$ & $\ldots$ & $\ldots$ \\
\hline K6 & $26 \pm 14$ & $1.51 \pm 0.16$ & $22 \pm 6$ & $0.68 \pm 0.31$ & $-9 \pm 3$ & $34 \pm 30$ \\
\hline K5 & $\sim 22$ & $\sim 2.31$ & $\sim 47$ & $\sim 1.28$ & $\ldots$ & $\ldots$ \\
\hline \multicolumn{7}{|c|}{6 April 1997} \\
\hline Core & $184 \pm 11$ & 0.00 & 0 & $<0.18$ & $-6 \pm 4$ & $2.7 \pm 1.4$ \\
\hline K9 & $47 \pm 11$ & $0.20 \pm 0.05$ & $67 \pm 45$ & $<0.35$ & $5 \pm 3$ & $4.9 \pm 2.4$ \\
\hline K8 & $171 \pm 28$ & $0.92 \pm 0.03$ & $77 \pm 2$ & $0.69 \pm 0.06$ & $\ldots$ & $\ldots$ \\
\hline K6 & $43 \pm 24$ & $1.50 \pm 0.17$ & $23 \pm 8$ & $0.79 \pm 0.20$ & $4 \pm 3$ & $36 \pm 29$ \\
\hline K5 & $\sim 19$ & $\sim 2.41$ & $\sim 46$ & $\sim 0.93$ & $\sim-2$ & $\sim 86$ \\
\hline $\mathrm{KE}_{1}$ & $\sim 18$ & $\sim 4.08$ & $\sim 64$ & $\sim 1.09$ & $\ldots$ & $\ldots$ \\
\hline \multicolumn{7}{|c|}{18 October 1997} \\
\hline Core & $244 \pm 6$ & 0.00 & 0 & $<0.13$ & $18 \pm 3$ & $5.2 \pm 0.5$ \\
\hline K9 & $43 \pm 8$ & $0.19 \pm 0.03$ & $85 \pm 17$ & $<0.21$ & $38 \pm 4$ & $6.0 \pm 1.1$ \\
\hline $\mathrm{K} 8$ & $107 \pm 16$ & $0.89 \pm 0.06$ & $76 \pm 5$ & $0.69 \pm 0.05$ & $\ldots$ & $\ldots$ \\
\hline K6 & $34 \pm 20$ & $1.52 \pm 0.23$ & $23 \pm 11$ & $0.79 \pm 0.35$ & $\ldots$ & $\ldots$ \\
\hline K5 & $\sim 25$ & $\sim 2.36$ & $\sim 42$ & $\sim 1.08$ & $\ldots$ & $\ldots$ \\
\hline $\mathrm{KE}_{1}$ & $\sim 20$ & $\sim 3.87$ & $\sim 65$ & $\sim 1.37$ & $\ldots$ & $\ldots$ \\
\hline \multicolumn{7}{|c|}{ 27 February 1999} \\
\hline Core & $414 \pm 9$ & 0.00 & 0 & $<0.19$ & $-42 \pm 3$ & $0.8 \pm 0.4$ \\
\hline K9 & $76 \pm 18$ & $0.24 \pm 0.03$ & $85 \pm 23$ & $0.25 \pm 0.11$ & $\ldots$ & $\ldots$ \\
\hline K8 & $33 \pm 28$ & $0.95 \pm 0.14$ & $85 \pm 10$ & $0.49 \pm 0.24$ & $\ldots$ & $\ldots$ \\
\hline $\mathrm{KE}_{2}$ & $\sim 60$ & $\sim 1.80$ & $\sim 42$ & $\sim 1.73$ & $\ldots$ & $\ldots$ \\
\hline \multicolumn{7}{|c|}{ 1 September 1999} \\
\hline Core & $345 \pm 11$ & 0.00 & 0 & $0.09 \pm 0.02$ & $-32 \pm 3$ & $2.1 \pm 0.6$ \\
\hline K9 & $145 \pm 14$ & $0.32 \pm 0.02$ & $75 \pm 11$ & $0.26 \pm 0.10$ & $-29 \pm 3$ & $7.3 \pm 1.7$ \\
\hline K8 & $24 \pm 18$ & $0.87 \pm 0.15$ & $83 \pm 14$ & $0.51 \pm 0.26$ & $\ldots$ & $\ldots$ \\
\hline $\mathrm{KE}_{2}$ & $\sim 47$ & $\sim 2.31$ & $\sim 49$ & $\sim 2.06$ & $\ldots$ & $\ldots$ \\
\hline \multicolumn{7}{|c|}{20 May 2000} \\
\hline Core & $520 \pm 7$ & 0.00 & 0 & $0.12 \pm 0.01$ & $-52 \pm 3$ & $1.7 \pm 0.3$ \\
\hline K9 & $134 \pm 19$ & $0.29 \pm 0.02$ & $81 \pm 5$ & $0.31 \pm 0.07$ & $-78 \pm 3$ & $3.3 \pm 1.6$ \\
\hline K8 & $92 \pm 16$ & $0.76 \pm 0.07$ & $75 \pm 6$ & $0.61 \pm 0.09$ & $\ldots$ & $\ldots$ \\
\hline $\mathrm{KE}_{2}$ & $\sim 42$ & $\sim 2.91$ & $\sim 57$ & $\sim 2.27$ & $\ldots$ & $\ldots$ \\
\hline
\end{tabular}

${ }^{1}$ Uncertain identification of this model component.

\subsection{Flux density evolution of model components}

Figure 9 presents the $5 \mathrm{GHz}, 8.4 \mathrm{GHz}, 15 \mathrm{GHz}$ and $22 \mathrm{GHz}$ total flux density evolution for the core and components $i 10$, $i 9, i 8, i 6$ and $i 5$ during the time span covered by our observations. Components not included in this figure, $\mathrm{X} 5_{1} ; i 4 ; i 3$ and $i 1$, showed little $8.4 \mathrm{GHz}$ total flux density evolution with weighted means of $(29 \pm 4) \mathrm{mJy},(17 \pm 4) \mathrm{mJy}([17 \pm 8] \mathrm{mJy}$ at $15 \mathrm{GHz})$, $(16 \pm 2) \mathrm{mJy}([9 \pm 5] \mathrm{mJy}$ at $15 \mathrm{GHz})$ and $(11 \pm 3) \mathrm{mJy}$, respectively.

This figure shows that the total flux density of $i 6$ at $8.4 \mathrm{GHz}$ decreased from $\sim 160 \mathrm{mJy}$ at the beginning of 1996 to $\sim 10 \mathrm{mJy}$ at the end of 1999, while the nearby feature $i 5$ displayed a quite stable total flux density of $(35 \pm 6) \mathrm{mJy}$ at $8.4 \mathrm{GHz}$ and $(20 \pm 5) \mathrm{mJy}$ 


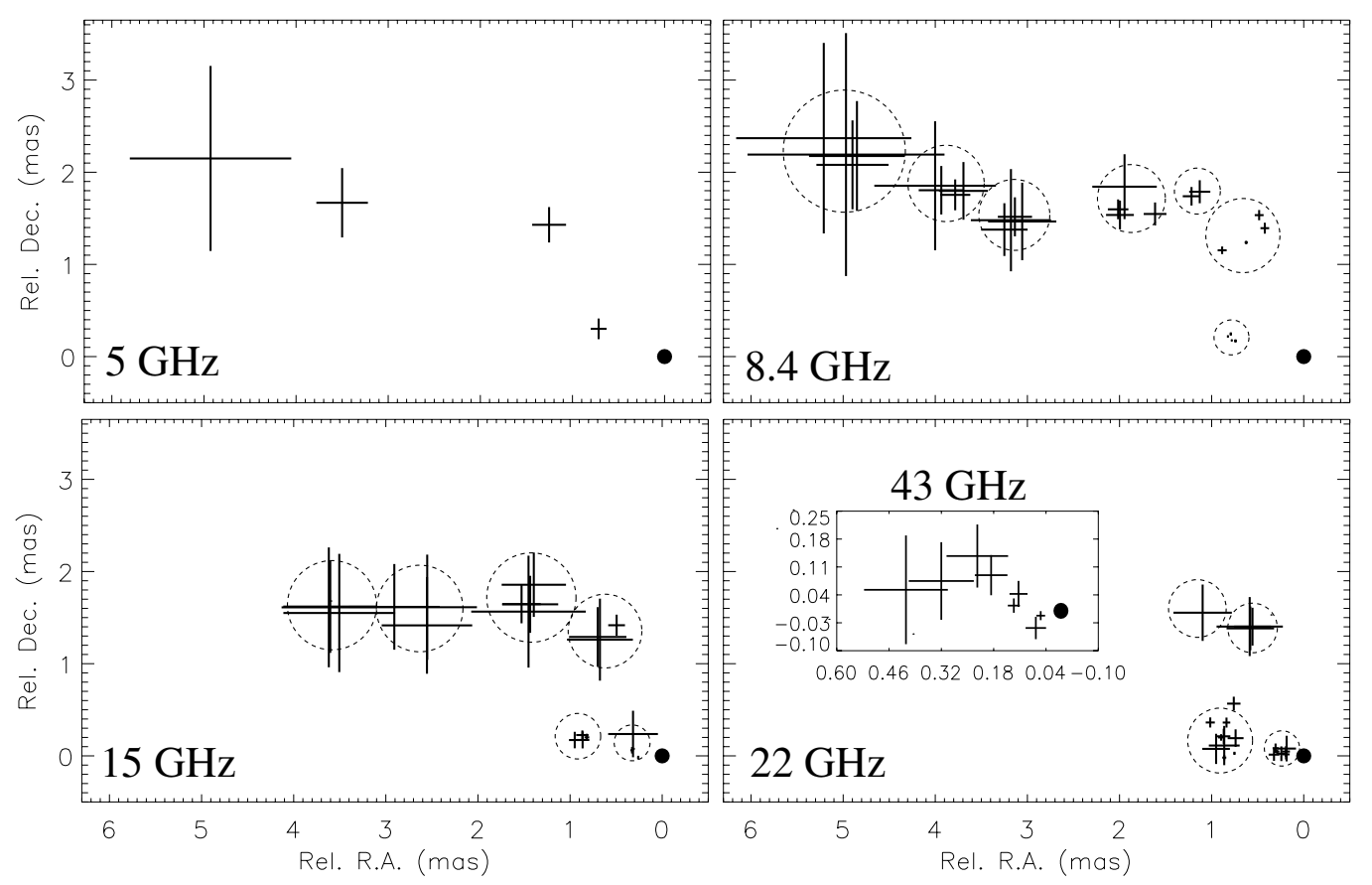

Fig. 6. Component positions and corresponding uncertainties (symbolized by the size of the crosses) for the model fits presented in Tables 2 to 6 . The dashed circles mark the various component clumps observed at each frequency. Components with large uncertainties in the fit parameters (for which no errors are given in Tables 2 to 6) and those with an uncertain identification (see Tables 4 and 5) are not plotted.

Table 6. $43 \mathrm{GHz}$ model fits.

\begin{tabular}{|c|c|c|c|c|c|c|}
\hline Comp. & $\begin{array}{c}I \\
(\mathrm{mJy}) \\
\end{array}$ & $\begin{array}{c}r \\
\text { (mas) }\end{array}$ & $\begin{array}{c}\theta \\
\left({ }^{\circ}\right) \\
\end{array}$ & $\begin{array}{c}F W H M \\
\text { (mas) }\end{array}$ & $\begin{array}{l}\chi \\
\left(^{\circ}\right) \\
\end{array}$ & $\begin{array}{l}m \\
\% \\
\end{array}$ \\
\hline \multicolumn{7}{|c|}{27 February 1999} \\
\hline Core & $315 \pm 12$ & 0.00 & 0 & $0.07 \pm 0.01$ & $\ldots$ & $\ldots$ \\
\hline Q10 & $68 \pm 9$ & $0.08 \pm 0.03$ & $123 \pm 13$ & $<0.22$ & $\ldots$ & $\ldots$ \\
\hline Q91 & $62 \pm 27$ & $0.21 \pm 0.02$ & $64 \pm 14$ & $0.21 \pm 0.04$ & $\ldots$ & $\ldots$ \\
\hline $\mathrm{Q} 9_{2}$ & $\sim 7$ & $\sim 0.40$ & $\sim 98$ & $\sim 0.02$ & $\ldots$ & $\ldots$ \\
\hline Q8 1 & $\sim 5$ & $\sim 0.62$ & $\sim 92$ & $\sim 0.10$ & $\ldots$ & $\ldots$ \\
\hline $\mathrm{Q}_{2}$ & $\sim 11$ & $\sim 0.79$ & $\sim 75$ & $\sim 0.28$ & $\ldots$ & $\ldots$ \\
\hline $\mathrm{Q}_{3}$ & $\sim 8$ & $\sim 1.08$ & $\sim 86$ & $\sim 0.22$ & $\ldots$ & $\ldots$ \\
\hline \multicolumn{7}{|c|}{ 1 September 1999} \\
\hline Core & $257 \pm 14$ & 0.00 & 0 & $<0.08$ & $\ldots$ & $\ldots$ \\
\hline Q10 & $79 \pm 18$ & $0.12 \pm 0.02$ & $70 \pm 21$ & $<0.20$ & $\ldots$ & $\ldots$ \\
\hline Q991 & $43 \pm 29$ & $0.26 \pm 0.06$ & $58 \pm 14$ & $<0.34$ & $\ldots$ & $\ldots$ \\
\hline $\mathrm{Q} 9{ }_{2}$ & $59 \pm 43$ & $0.42 \pm 0.10$ & $83 \pm 19$ & $0.29 \pm 0.22$ & $\ldots$ & $\ldots$ \\
\hline \multicolumn{7}{|c|}{20 May 2000} \\
\hline Core & $252 \pm 12$ & 0.00 & 0 & $0.07 \pm 0.04$ & $-51 \pm 7$ & $1.4 \pm 0.5$ \\
\hline Q11 & $163 \pm 9$ & $0.06 \pm 0.01$ & $103 \pm 25$ & $<0.08$ & $-59 \pm 8$ & $1.4 \pm 0.6$ \\
\hline Q10 & $82 \pm 12$ & $0.13 \pm 0.01$ & $84 \pm 11$ & $<0.21$ & $-74 \pm 8$ & $3.1 \pm 1.1$ \\
\hline Q9 & $162 \pm 34$ & $0.33 \pm 0.08$ & $77 \pm 16$ & $0.47 \pm 0.07$ & $\ldots$ & $\ldots$ \\
\hline Q8 1 & $\sim 14$ & $\sim 0.76$ & $\sim 71$ & $\sim 0.20$ & $\ldots$ & $\ldots$ \\
\hline $\mathrm{Q}_{2}$ & $\sim 18$ & $\sim 1.10$ & $\sim 81$ & $\sim 0.32$ & $\ldots$ & $\ldots$ \\
\hline
\end{tabular}

at $15 \mathrm{GHz}$; $i 5$ was still detected in May 2000, whereas $i 6$ had become undetectable by that time.

A similar trend as for $i 6$ was observed for component $i 8$ at the first bend, which quasi-monotonically decreased its total flux density by $\sim 500 \mathrm{mJy}$ at $8.4 \mathrm{GHz}$ during the same period. Simultaneously, the total flux density of the nearby component $i 9$ remained almost constant at $\sim 100 \mathrm{mJy}$ from 1996 to 1999. Component $i 10$ likewise maintained a very stable total flux density of $(74 \pm 7) \mathrm{mJy}$ at $43 \mathrm{GHz}$ from February 1999 to May 2000.

While the total flux density evolution of $i 8, i 9$ and $i 10$ was monotonic for most of the time between 1996 and 2000, the core

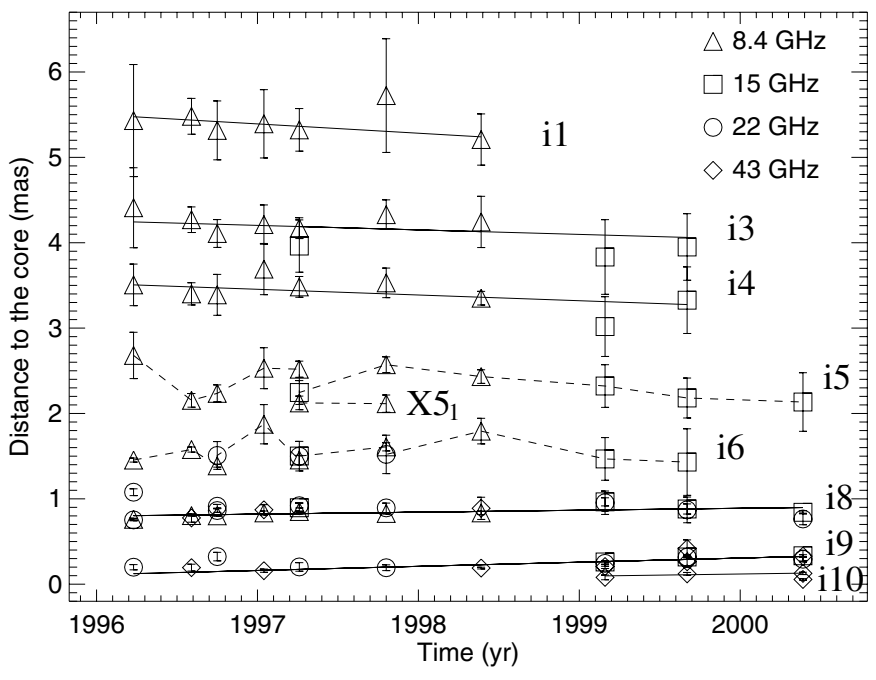

Fig. 7. Distance to the core as a function of time for the innermost (within $\$ 7$ mas) model components at $8.4 \mathrm{GHz}, 15 \mathrm{GHz}, 22 \mathrm{GHz}$ and $43 \mathrm{GHz}$. Note that, for the sake of clarity, only components observed during more than two epochs have been plotted. The epochs used are those in Tables 3 to 6, as well as August 1996, January 1997 and May 1998 from Gómez et al. (2001). Linear fits of the radial positions for some model components are also shown (solid lines). The dashed lines connect the radial positions of components $i 5, \mathrm{X} 5_{1}$ and $i 6$. Components with large uncertainties in the fit parameters (for which no errors are given in Tables 2 to 6) and those with an uncertain identification (see Tables 4 and 5) are not plotted.

displayed significant rapid fluctuations on typical time-scales of several weeks and with amplitudes of $\sim 20 \%$ around $400 \mathrm{mJy}$.

Note that, whereas the total flux density of the core fluctuated and the flux densities of $i 9$ and $i 10$ remained almost constant, the behaviour of $i 8$ coincided with the single-dish integrated total flux density evolution of $0735+178$ between early 1996 and late 


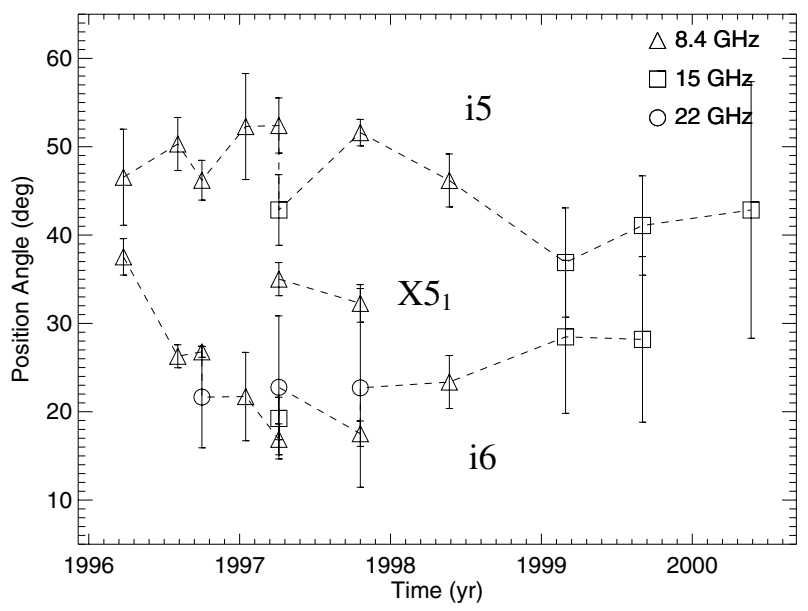

Fig. 8. Structural position angles as a function of time for components $i 5, \mathrm{X} 5_{1}$ and $i 6$. The points shown are the same as those in Fig. 7.

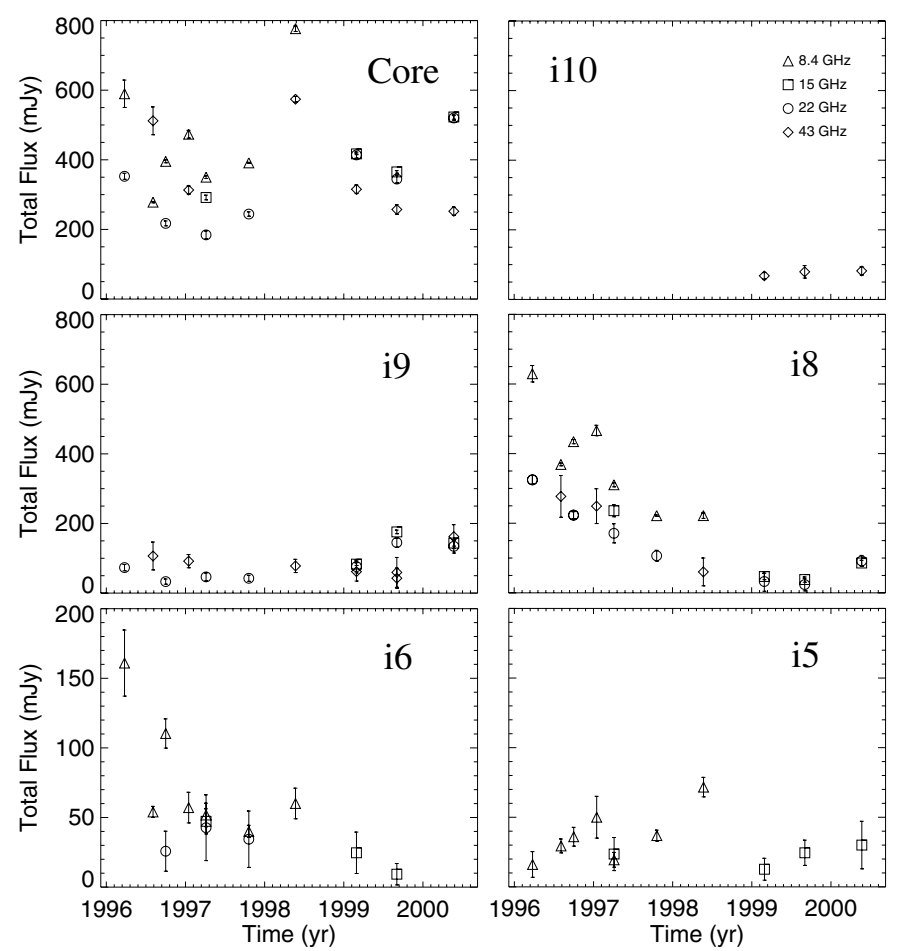

Fig. 9. $5 \mathrm{GHz}, 8.4 \mathrm{GHz}, 15 \mathrm{GHz}$ and $22 \mathrm{GHz}$ and $43 \mathrm{GHz}$ total flux density evolution for the Core, $i 10, i 9, i 8, i 6$ and $i 5$ components. The epochs shown are those in Table 1, as well as August 1996, January 1997 and May 1998 from Gómez et al. (2001). Each frequency is represented by a different symbol, as indicated in the right top inset. Note the different total flux density scale used for the plots of $i 6$ and $i 5$.

1997 at $4.8 \mathrm{GHz}, 8 \mathrm{GHz}$ and $14.5 \mathrm{GHz}$ (Aller et al. 1999) and at $22 \mathrm{GHz}$ and $37 \mathrm{GHz}$ (Fig. 10 and Teräsranta et al. 2004). The data from both single dish monitoring programs showed a monotonic decrease in total flux density of $\sim 500 \mathrm{mJy}$ at all frequencies during this time range, which is precisely the amount of flux density decrease observed for $i 8$.

This, together with the fact that $i 8, i 9, i 10$ and the core are typically responsible for more than the $90 \%$ of the total emission of the source at high frequencies $(22 \mathrm{GHz}$ and $43 \mathrm{GHz}$ ) and more than $80 \%$ at lower frequencies, shows that the decrease in total flux density measured by the single-dish monitoring was determined primarily by the flux density decrease of $i 8$.

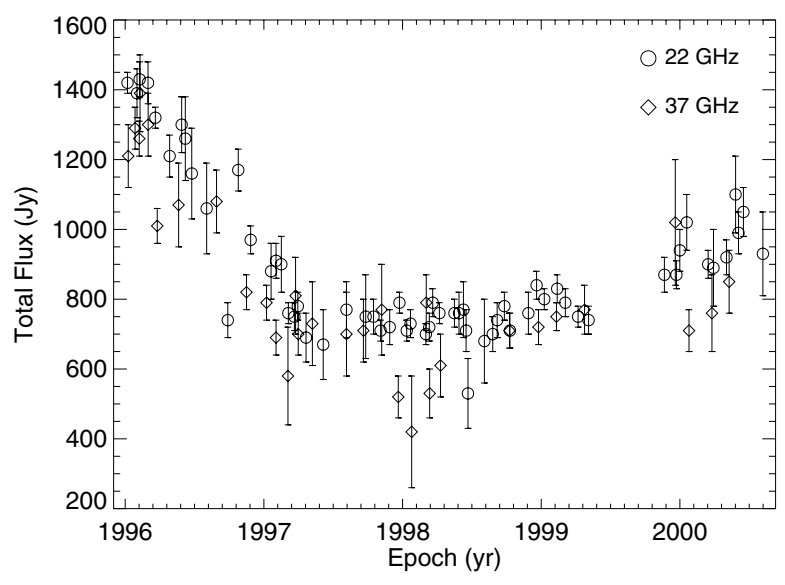

Fig. 10. Integrated $22 \mathrm{GHz}$ and $37 \mathrm{GHz}$ total flux density light curves of $0735+178$ from 1996 to 2000 . Data obtained by the monitoring program of extragalactic radio sources of the Metsähovi radio observatory (Teräsranta et al. 2004).

\subsection{Doppler factor and geometry of the inner jet}

The highly bent VLBI structure of $0735+178$ suggests that the source is oriented at a small angle to our line of sight. This is also supported by the large superluminal motions measured in the jet before 1993, and by the observed high degree of variability for the core (see Sect. 4.2).

It is possible to constrain this angle using our proper motion measurements and previous estimates of the jet's Doppler factor $^{5}(\delta)$. Güijosa \& Daly (1996) computed a $\delta_{\text {eq }} \approx 7$ (estimation of the Doppler factor under the assumption that the magnetic field and the relativistic particles of the plasma are in equipartition). Using different assumptions, Lähteenmäki \& Valtaoja (1999) reported $\delta_{\text {var }} \approx 4$ (Doppler factor estimated from the variability of the long term radio light curves). Other estimates of $\delta$ for $0735+178$ in the literature lie between the previous values of $\delta_{\text {eq }}$ and $\delta_{\text {var }}$. To test these values, we have computed the variability Doppler factor $\delta_{\text {var }}$ of the core in $0735+178$ through the expression defined by Jorstad et al. (2005) and using our own data. The result gives $\delta_{\mathrm{var}} \approx 5$, which agrees well with the previous estimates.

We then assume that the true $\delta$ for the innermost jet in $0735+178$ ranges between 4 and 7 , and use our observed proper motions of components $i 8, i 9$ and $i 10$ (which essentially range between $0.1 c$ and $1.3 c$ ) to estimate the possible ranges of viewing angles $(\theta)$ and plasma bulk Lorentz factors $(\Gamma)$ for the first mas of the jet, using the relation $\beta_{\text {ap }}=(\beta \sin \theta) /(1-\beta \cos \theta)$ where $\beta_{\text {ap }}$ is the measured apparent proper motion (e.g., Pearson $\&$ Zensus 1987) together with the definition of the Doppler factor (see above). This constrains $\theta$ to values between $0^{\circ}$ and $9^{\circ}$, as small as expected, and constrains $\Gamma$ to values between 2 and 4 . Note that the speeds used for these estimates are associated with jet features that are related to bends (at least for $i 8$; see Figs. 4 and 5) and we have not observed fast superluminal speeds such as those reported before 1993. Therefore, our estimates of $\theta$ and $\Gamma$ should be related to the pattern speeds of the innermost bent jet structure.

${ }^{5} \delta=[\Gamma(1-\beta \cos \theta)]^{-1}$ where $\Gamma=\left(1-\beta^{2}\right)^{-1 / 2}$ is the Lorentz factor, $\beta$ is the speed in units of the speed of light and $\theta$ is the angle between the direction of the flow and the line of sight. 

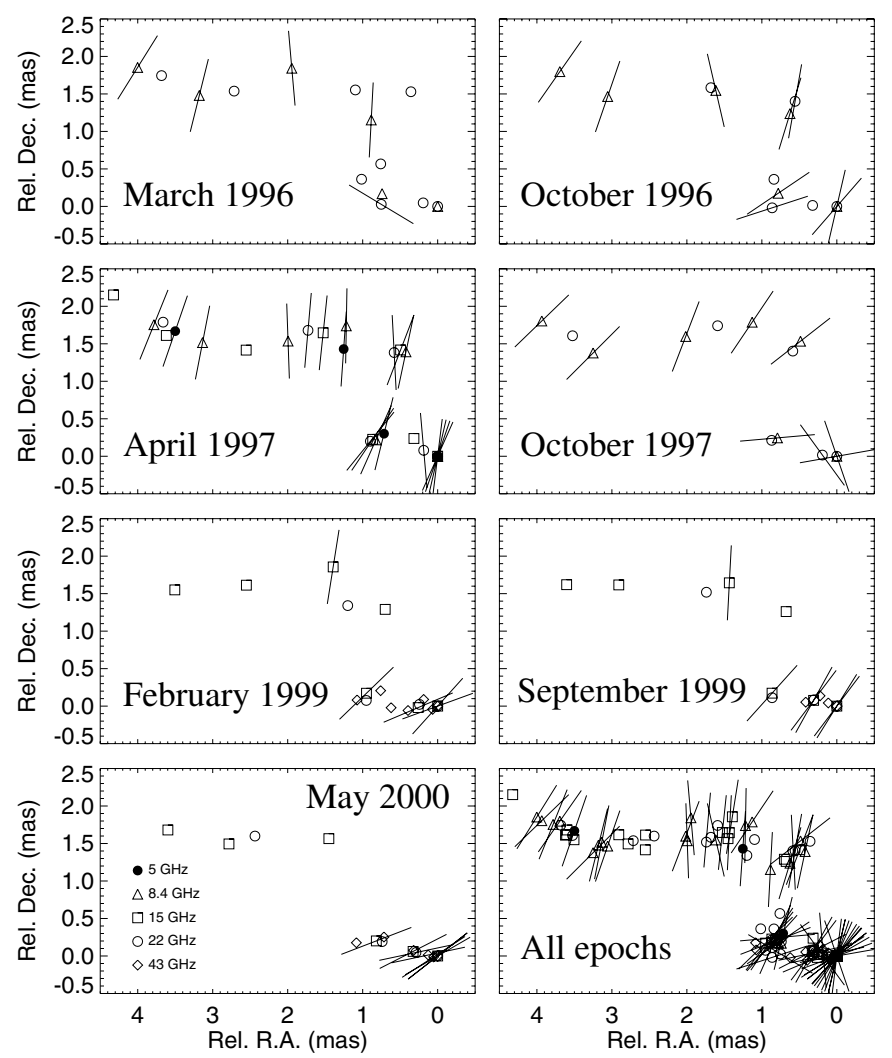

Fig. 11. Time-dependent $\chi$ distribution at the location of the model fit components from Tables 2 to 6 . The lines represent the orientation of $\chi$, whereas the observing frequency for each component is shown by a different symbol (as indicated in the left bottom panel).

\subsection{Polarization evolution of model components}

Figure 11 represents the $\chi$ values at the locations of the model fit components in Tables 2 to 6 . In agreement with Gómez et al. (1999), this figure shows that the polarization electric vectors were predominantly perpendicular to the local direction of the northern jet (outwards from the location of i6) at all the observing epochs.

For the region between the core and the first strong bend, $\chi$ shows a clear time dependence, which is more clearly shown by the plots of $\chi$ as a function of time for $i 8$ and the core (Fig. 12). Note that the $\chi$ behaviour of $i 9$ is very similar to that of the core. This is because these two components belong to the same polarization region, although the model fits for the total intensity jet are able to separate them. While the core shows irregular polarization variability from 1996 to 2000 (as is expected from its high level of total flux density variability), the $\chi$ for $i 8$ linearly increases at all frequencies between $\sim-120^{\circ}$ in March 1996 to $\sim-30^{\circ}$ in April 1997. Furthermore, it experienced an abrupt rotation by $\sim-60^{\circ}$ in October 1997 and then maintained a much more stable value of $\sim-45^{\circ}$ during the last three observing epochs, with a small decrease to $\sim-25^{\circ}$ observed in May 2000.

The $\sim 90^{\circ}$ monotonic rotation of $i 8$, from 1996 to 1998 , shows evidence of correlation with its total flux density evolution and also with the integrated total flux density evolution of $0735+178$ (see Sect. 4.2) during the same time range. This rotation can not be produced by time dependent Faraday rotation, which would progressively change the relative $\chi$ orientation of i8 for the different frequencies. A possible explanation for this behaviour could be either the passage of a shock through the

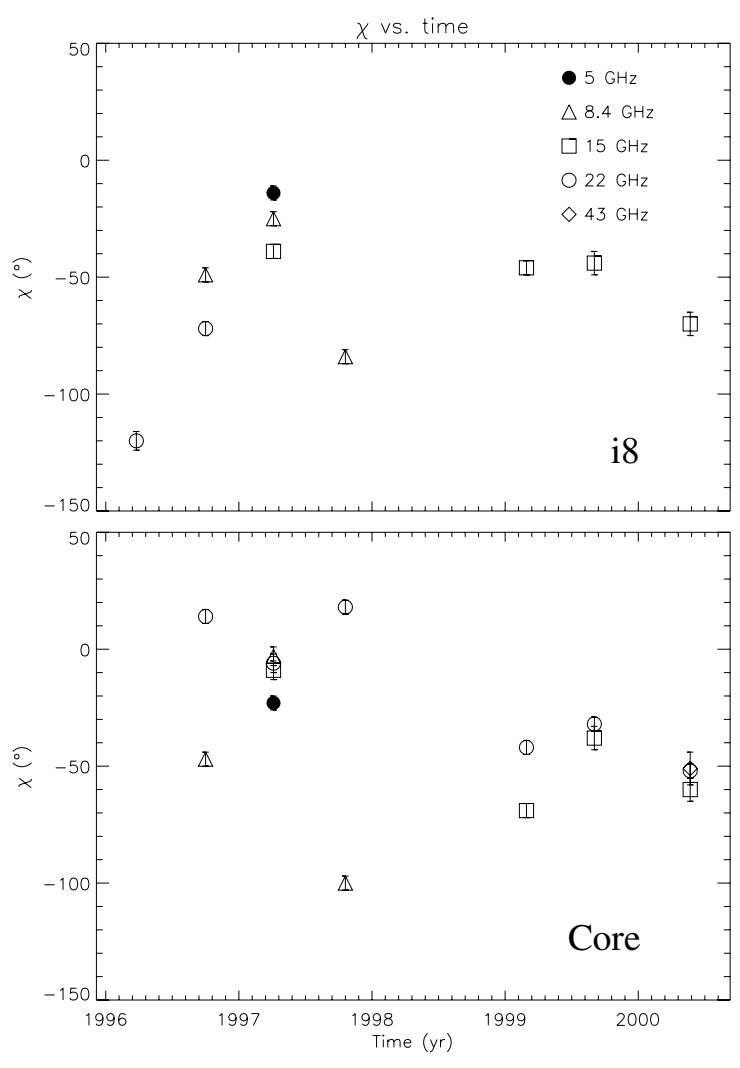

Fig. 12. $\chi$ as a function of time for $i 8$ (top) and the core (bottom). The observing frequency for each component is represented by a different symbol, as indicated in the top plot.

first bend or the interaction of this bend with a clumpy external medium, as proposed by Gabuzda et al. (2001), see also Sect. 1. We favour the jet-external medium interaction since, although i8 moved outward from the core, it did so with a slow subluminal speed not typical of moving shocks in relativistic jets and it displayed a stable position and seemed to be well "attached" to the first bend of the underlying jet. In addition, a shock passing through the first bend would be expected to produce a clockwise rotation of $\chi$ (for a plane shock perpendicular to the jet axis and moving following the jet bending towards the north), contrary to what was shown by our observations.

Note also that the behaviour of $i 8$ is quite different from that of $i 6$, which maintained a stable $\chi$ orientation during the time covered by our observations, although its total flux density monotonically decreased (as for $i 8$ ).

\section{Summary and conclusions}

We have investigated the kinematic, total and polarized flux density evolution of roughly the inner 6 mas of the VLBI jet in $0735+178$ from 1996 to 2000 .

Components $i 1$, i3 and $i 4$ displayed essentially stationary positions during the time spanned by our observations. They also have stable $I$ and $P$ flux densities and $\chi$ values. This suggests that the region in which these components are located is mainly composed of the quiescent underlying jet, whose dynamical timescales seem to be longer than those covered by our observations.

For the innermost jet, we have found subluminal or slow superluminal proper motions with apparent speeds of $(0.58 \pm$ $0.10) c,(1.21 \pm 0.10) c$ and $(0.65 \pm 0.53) c$ for components $i 8, i 9$ and $i 10$, respectively. The variability of the total flux density of the core allowed us to estimate its Doppler factor $\delta \approx 5$. Using these measurements and previous estimates of the jet Doppler 
factor, we have computed the possible values for the Lorentz factor $\Gamma$, which ranged from 2 to 4 , and the angle to the line of sight $\theta$ to be $\leq 9^{\circ}$. As we have not detected fast superluminal motions, such as those reported for this source before 1993, and the apparent speeds used to estimate $\theta$ and $\Gamma$ were based on the slow proper motions of jet features near the jet bends, our estimates of $\Gamma$ should be related to the typical pattern speeds of the innermost bent jet structure. Note that Lorentz factors in bent regions similar to those estimated for $0735+178$ have been shown by three-dimensional numerical simulations of relativistic helical jets (Aloy et al. 2003).

Therefore, with the exception of $i 6$ (whose position changed significantly, moving first north and then back south, leading to a decrease in the curvature of the second bend of the jet), all the jet components traced during the time range covered by our monitoring were either quasi-stationary or showed slow motions, contrary to the situation observed before 1993 when $7 c \leq \beta_{\text {ap }} \leq 12 c$ (Gabuzda et al. 1994, and references therein).

A possible explanation for this discrepancy was suggested by Gómez et al. (2001), who proposed a scenario in which the jet experienced different regimes before 1993 and after 1995. Before 1993, rapid superluminal motions $\left(7 c \leq \beta_{\text {ap }} \leq 12 c\right)$ were reported, the structure of the jet remained mainly rectilinear (Gabuzda et al. 1994 and Gómez et al. 2001) and the radio flux densities from the UMRAO and Metsähovi monitoring programs were the highest ever detected in $0735+178$ (up to $\sim 5 \mathrm{Jy}$, see Aller et al. 1999 and Teräsranta et al. 2004). This could be called an "active" regime of the jet in $0735+178$. From 1995 to 2000 , in the "quiescent" regime, only slow motions were detected (see Sect. 4.1), the source showed a highly curved structure with two sharp bends and its integrated flux densities between $5 \mathrm{GHz}$ and $37 \mathrm{GHz}$ decreased roughly linearly up to $\sim 1997$ to $\sim 1998$, reaching its lowest known values $(\$ 1$ Jy, Aller et al. 1999 and Teräsranta et al. 2004).

This scenario is supported by the $15 \mathrm{GHz}$ VLBA images of $0735+178$ obtained by the MOJAVE program ${ }^{6}$ for epochs after November 2002 (Lister \& Homan 2005). In agreement with the softening of the second bend during the time covered by our observations, these new images show a fairly rectilinear jet structure oriented to the north-east (as reported for early VLBI images before 1993). The typical double-bend structure displayed by $0735+178$ between 1995 and 2000 is no longer evident. In addition, the $15 \mathrm{GHz}$ integrated total flux density of the source in November 2002 increased by $100 \%$ with respect to our previous observation at $15 \mathrm{GHz}$ (in May 2000), which seems to be accompanied by the appearance of rapid superluminal motions within the innermost four mas of the jet.

In addition, the optical ( $V, R$ and $I$ band) light curves of 0735+178 from January 1995 to March 2001 reported by Qian $\&$ Tao (2004) also agree with the proposed scenario. The source was observed to decrease its optical flux density from 1995 to January 1998, when it reached one of its faintest levels ever observed (with a magnitude in $V, m_{V}=16.68$ ). After that, $0735+178$ became brighter again to reach a new maximum in February $2001\left(m_{V}=14.54\right)$. This behaviour is consistent with the one showed by the radio light curves in Fig. 10. Hanski et al. (2002), through the analysis of a different optical data set from 1988 to 1998 together with the radio light curves from the Metsähovi monitoring program, showed that the optical flux density level was always high for $0735+178$ when the modelled radio flux density was at the peak of a flare.
Confirmation of superluminal motions from the data of the MOJAVE program will provide added support for the proposed bimodal scenario. We could therefore consider the possibility of a cyclic behaviour for $0735+178$ in which periods of enhanced activity, with ejection of superluminal components, are followed by epochs of low activity, with a highly twisted jet geometry, and vice-versa. During the high activity period the ejection of superluminal components, associated with strong shocks, would lead to an increase of the broad band emission of the source, enhanced by the large Doppler boosting factors expected for this source given its small viewing angle. An increase in the momentum of the component may lead to a more rectilinear, quasiballistic, motion of the component, and therefore for the apparent jet geometry. Subsequent low activity periods could reveal the quiescent jet, characterized by a highly twisted geometry. This would most probably require a jet with a changing direction of ejection, since otherwise we would expect the quiescent jet to follow the straight funnel left by the previous period of high activity.

Acknowledgements. We gratefully acknowledge Harri Teräsranta for providing data from the Metsähovi monitoring of AGN in advance of publication. We gratefully acknowledge Margo F. Aller and Hugh D. Aller for providing data from the University of Michigan Radio Astronomy Observatory, which is supported by funds of the University of Michigan and Barry Clark for providing ad hoc VLA time to determine the absolute $\chi$ of the data. We thank Alan L. Roy for his helpful suggestions and Esther Sánchez for preparing the figures and tables. We are also grateful to the anonymous referee for his/her useful comments. I. Agudo acknowledges financial support from the EU Commission through HPRN-CT-2002-00321 project (ENIGMA network). This research has been supported by the Spanish Ministerio de Educación y Ciencia and the European Fund for Regional Development through grant AYA2004-08067-C03-03. The work at Boston University was supported by National Science Foundation grant AST0406865 .

\section{References}

Agudo, I., Gómez, J. L., Martí, J. M., et al. 2001, ApJ, 549, L183 Aller, M. F., Aller, H. D., Hughes, P. A., et al. 1999, ApJ, 512, 601 Aloy, M. A., Martí, J. M., Gómez, J. L., et al. 2003, ApJ, 585, L109 Carswell, R. F., Strittmatter, P. A., Williams, R. D., et al. 1974, ApJ, 190, L101 Fomalont, E. 1981, Newsletter. NRAO, 3, 3

Gabuzda, D. C., Wardle, J. F. C., Roberts, D. H., et al. 1994, ApJ, 435, 128 Gabuzda, D. C., Gómez, J. L., \& Agudo, I. 2001, MNRAS, 328, 719 Gómez, J. L., Marscher, A. P., Alberdi, A., et al. 1999, ApJ, 519, 642 Gómez, J. L., Marscher, A. P., Alberdi, A., et al. 2000, Science, 289, 2317 Gómez, J. L., Guirado, J. C., Agudo, I., et al. 2001, MNRAS, 328, 873 Güijosa, A., \& Daly, R. A. 1996, ApJ, 461, 600

Hanski, M. T., Takalo, L. O., \& Valtaoja, E. 2002, A\&A, 394, 17 Homan, D. C., Ojha, R., Wardle, J. F. C., et al. 2001, ApJ, 549, 840 Jorstad, S. G., Marscher, A. P., Lister, M. L., et al. 2005, AJ, 130, 1418 Kellermann, K. I., Vermeulen, R. C., Zensus, J. A., et al. 1998, AJ, 115, 1295 Kellermann, K. I., Lister, M. L., Homan, D. C., et al. 2004, ApJ, 609, 539 Kollgaard, R. I., Wardle, J. F. C., Roberts, D. H., et al. 1992, AJ, 104, 1687 Lähteenmäki, A., \& Valtaoja, E. 1999, ApJ, 521, 493

Leppänen, K. J., Zensus, J. A., \& Diamond, P. J. 1995, AJ, 110, 2479 Lister, M. L., \& Homan, D. C. 2005, AJ, 130, 1389

Lovell, J. 2000, in Proceedings of the VSOP Symposium, Astrophysical Phenomena Revealed by Space VLBI, ed. H. Hirobayashi, P. G. Eduards, \& D. W. Murphy, 301

Ojha, R., Homan, D. C., Roberts, D. H., et al. 2004, ApJS, 150, 187

Pearson, T. J., \& Zensus, J. A. 1987, in Superluminal Radio Sources, ed. J. A. Zensus, \& T. Pearson (Cambridge Univ. Press), 1

Pearson, T. J., Shepherd, M. C., Taylor G. B., et al. 1994, AAS, 185, 808

Qian, B., \& Tao, J. 2004, PASP, 116, 161

Rector, T. A., \& Stocke, J. D. 2001, ApJ, 122, 565

Teräsranta, H., Achren, J., Hanski, M., et al. 2004, A\&A, 427, 769

Ulvestad, J. S., Johnston, K. J., \& Weiler, K. W. 1983, ApJ, 266, 18 